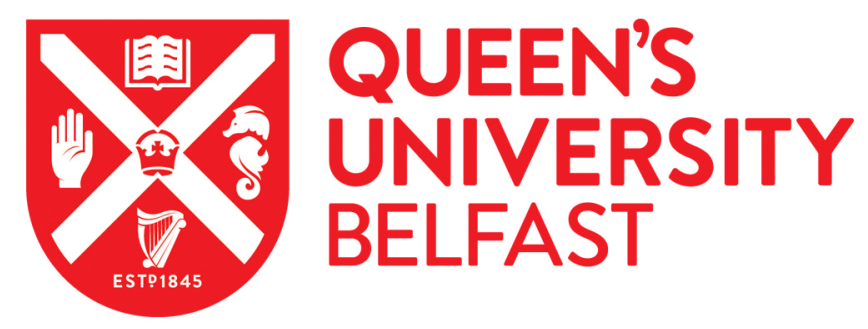

\title{
Adaptive hierarchical sliding mode control for full nonlinear dynamics of uncertain ridable ballbots under input saturation
}

Do, V-T., Lee, S-G., \& Van, M. (2021). Adaptive hierarchical sliding mode control for full nonlinear dynamics of uncertain ridable ballbots under input saturation. International journal of robust and nonlinear control, 31(8), 2882-2904. https://doi.org/10.1002/rnc.5423

Published in:

International journal of robust and nonlinear control

Document Version:

Peer reviewed version

Queen's University Belfast - Research Portal:

Link to publication record in Queen's University Belfast Research Portal

Publisher rights

(c) 2021 John Wiley \& Sons, Ltd

This work is made available online in accordance with the publisher's policies. Please refer to any applicable terms of use of the publisher.

\section{General rights}

Copyright for the publications made accessible via the Queen's University Belfast Research Portal is retained by the author(s) and / or other copyright owners and it is a condition of accessing these publications that users recognise and abide by the legal requirements associated with these rights.

Take down policy

The Research Portal is Queen's institutional repository that provides access to Queen's research output. Every effort has been made to ensure that content in the Research Portal does not infringe any person's rights, or applicable UK laws. If you discover content in the Research Portal that you believe breaches copyright or violates any law, please contact openaccess@qub.ac.uk. 


\title{
Adaptive Hierarchical Sliding Mode Control for Full Nonlinear Dynamics of Uncertain Ridable Ballbots under Input Saturation
}

\author{
Van-Thach Do ${ }^{1}$, Soon-Geul Lee ${ }^{1 *}$, Mien $\operatorname{Van}^{2}$
}

\footnotetext{
${ }^{1}$ Kyung Hee University, 1732, Deogyeong-daero, Giheung-gu, Yongin-si, Gyeonggi-do 17104, Republic of Korea. (thachdovan@khu.ac.kr, sglee@khu.ac.kr)

${ }^{2}$ School of Electronics, Electrical Engineering and Computer Science, Queen's University Belfast, Belfast United Kingdom of Great Britain and Northern Ireland BT7 1NN (e-mail: m.van@qub.ac.uk)

* Corresponding author.
}

\section{SUMMARY}

This article proposes a nonlinear adaptive controller based on a hierarchical sliding mode control framework to stabilize the operation of a ridable ballbot based on the full nonlinear dynamics (FND). The FND of ballbots is a multiple-input-multiple-output underactuated system, in which the input matrix is non-invertible. By applying a novel approach to transform the FND into two subsystems, the invertibility of the input matrix of these subsystems can be guaranteed. Thus, a hierarchical sliding mode control (HSMC) scheme can be applied to control the ridable ballbot system by stabilizing these subsystems. Moreover, we propose new adaptive laws to enhance the robustness of the HSMC to deal with the effects of parameter variations and exogenous disturbances on the modeled dynamics. Chattering and actuator saturation phenomena are also reduced or avoided under the proposed controller. Comparative simulations are conducted to demonstrate the merit features of the proposed approach and the robustness of the developed control algorithm.

Keywords: Underactuated system, sliding mode control, input saturation, chattering reduction, uncertain system.

\section{INTRODUCTION}

A ridable ballbot is a vehicle that has the same mechanical structure as a ballbot [1]-[4]. It is designed with a large size and robust mechanical structure such that a person can ride on the platform [5]. The ballbot is a multiple-input-multiple-output (MIMO), underactuated mechanical system (UMS) possessing five degrees of freedom. Due to the complication of dynamic structure and the natural instability of the system, designing controllers and finding proper sets of control gains for the system are of challenging processes. Moreover, the effects of parameter variation, disturbances, and input saturations of actuators reduce the tracking performances of the practical ballbot systems. In ridable ballbots, the system parameters (such as the system mass, the moment of inertia, and friction effects, etc.) are continuously changed due to the physical body of riders and the characteristics of the ground surface. Thus, the robustness property of the control system plays a pivotal role in ensuring operability of the system under various uncertain effects frominternal dynamics and exogenous environment. 
Control algorithms for ballbots have been developed and benchmarked in the past decades by linear, nonlinear, and intelligent approaches. In [1], [5]-[8] the robustness of linear controllers are obtained by integral actions. However, the control gains must be carefully selected to avoid the main drawbacks of the integral control that provide large overshoot and require large control efforts. In [9], a robust linear controller has been proposed for a ballbot, which is built using a LEGO Mindstorms kit. The parameter variation problem has been addressed in that research; however, the control gains are offline tuned by the LMI toolbox. Generally, the linear control approaches provide poor performance for time varying uncertainties and exogenous disturbance, which limits its practical applicability for uncertain nonlinear systems like ballbots. In [5], a passivity-based nonlinear controller has been proposed to govern the balance and transfer to an experimental ballbot. In [10], a double-loop controller, constructed from partial feedback linearization and proportional-integral controllers, has been designed for a ridable ballbot. However, the robustness of the controllers has not been thoroughly demonstrated in these studies. In [11], an intelligent controller has been proposed for a ballbot system by combining fuzzy and supervisory controllers. Its robustness is demonstrated only in station-keeping experiments. In [12], a backstepping sliding mode control has been proposed for a ballbot. By integrating sliding mode control and recurrent interval type-2 fuzzy neural networks, the robustness of the controller can be enhanced. However, the use of fuzzy neural networks in the control scheme design increases the computational burden of the system and the stability of the system is difficult to guarantee.

In order to enhance robustness for ballbot systems, many controllers have been proposed based on sliding mode control (SMC) and its advances so far. SMC is a well-known, robust, nonlinear control method. SMC approaches have been developed and applied for many nonlinear systems due to its strong robustness against matched disturbance (at least in theory) [13]-[17]. However, conventional sliding surfaces cannot be straightforwardly applied for underactuated systems [18], because of the existence of non-invertible input matrices. Several efforts have been made to reconstruct the conventional SMCs so that they can be applicable for UMSs. In [19]-[22], the dynamic model of an UMS is decoupled into actuated and underactuated subsystems. Then, the ultimate control input is derived based on a new defined sliding surface, which is constructed by the tracking error vectors of these subsystems. By applying a global change of coordinates in [23], the dynamic models of a class of UMSs with integrable normalized momentum (INM) are transformed into cascade normal forms. Then, SMC schemes can be designed to asymptotically stabilize the system [24]-[26]. In [27], a new SMC approach is developed to handle UMSs with non- INM by maintaining a subset of actuated variables on sliding manifolds. Then, the non-INM can be approximated by an INM in finite time. In [28], a fuzzy-based adaptive SMC is proposed for MIMO underactuated systems; however, a regularized inverse approximation for the control input matrix is required for constructing the equivalent control law. In [29], the dynamics of a ballbot is obtained by deriving a quaternion-based model using the Lagrangian approach. The non-invertibility of the input matrix is avoided by neglecting the quaternion scalar value and the translational dynamics. Thus, the robustness of the SMC for that system is guaranteed for the balance control only, i.e., for the stabilization of orientation angles. In [30], a combination of SMC and partial feedback linearization is proposed to govern the balance and transfer of a ridable ballbot. The controller is designed using the coupled ballbot dynamics without controlling the yaw motion. Other approaches are cascade SMCs [31] and hierarchical SMC (HSMC) schemes [18], [32]. However, the effects of parameter variations and external disturbance are not carefully considered and analyzed in these approaches. In addition, these algorithms are designed for single-input-multiple-output UMSs. The robustness of the conventional HSMC is enhanced by combining with intelligent adaptive laws such as fuzzy-based [33]-[35] or neural network-based approaches [36]-[38]. In [39]-[41], the aggregation of the HSMC scheme in [32] is applied to stabilize a ridable ballbot system. However, model uncertainties and external disturbances are not considered when deriving system dynamics and control scheme design. To address these issues, an integral backstepping HSMC is proposed for an experimental ballbot [42] with considering matched and mismatched uncertainties in the model dynamics. A new system stability is presented based on the special property of sliding surfaces (Lemma 2, [42]) of the ballbot. However, there exist constraints on the upper bound of matched uncertainties. These constraints can be relaxed by using a radial bias function neural network, which 
is used to estimate matched uncertainties in [36].

During the development of ballbots, controllers have been proposed based on four types of dynamic models: (i) decoupled two dimensional dynamics [1], [4], [5], [39], [40], [42]; (ii) coupled two vertical dynamics [30], [36], [41]; (iii) partly neglected dynamics [29]; and (iv) linearized full-nonlinear ballbot dynamics (FNBD) [7], [8], [43], [44]. Even though these approaches investigated incomplete-nonlinear dynamic models, robust controllers were proposed to handle model uncertainties and exogenous disturbance under small deviations of body-lean angles. Hence, the effectiveness of these approaches can be verified based on simulations or experimental results. However, the coupling effects between decoupled dynamics are neglected. Moreover, the assumptions of independent motion of decoupled planes must be made for decoupling the FNBD. These assumptions only happen under small deviations from the upright posture. Under larger tilt angles, the coupling effects significantly increase, and these assumptions might not be held. In addition, the controllers for linearized models are valid only for small angles, where linearized conditions hold. Thus, stabilizing the FNBD plays an important role, not only for governing the operation of ballbots but also addressing the control of UMS under strong coupling case. Moreover, designing robust nonlinear controllers for a full ballbot dynamics is desirable, especially for ridable ballbots, where nonlinear characteristics, variations of model parameters, and exogenous disturbance always exist during the operation of the ballbots. However, there are no nonlinear controllers that have been proposed for FNBD so far (without decoupling or partly neglecting the full dynamic model) for both numerical simulations and experiments.

Motivated by the above technological voids, in this study, we designed a robust, adaptive HSMC controller to stabilize a ridable ballbot system using the FNBD. The system operation is governed by three motors connected to three omnidirectional wheels. First, to tackle the non-invertibility of the input matrix of the FNBD, we proposed a novel approach to transform the full dynamics into two subsystems, where the invertibility of input matrices of these subsystems are ensured. This transformation is obtained by dividing the generalized coordinate vector into two sub-vectors, i.e., the pose and the orientation vectors. The pose vector describes the pose information of a mobile robot in the $x y$-plane, while the orientation vector describes the roll, pitch, and yaw angles of the ballbot body. By considering the effects of model uncertainties, exogenous disturbances, and the input saturation phenomenon, a new adaptive law is proposed to enhance the robustness of the conventional HSMC (CHSMC). In addition, an auxiliary system is designed to compensate for the input saturation, which commonly happens in practical ballbot systems.

The main contributions of this article are summarized as follows:

- To the best of our knowledge, this is the first nonlinear controller proposed for FNBD, which is a MIMO, UMS with strong input coupling [45].

- Based on observing the properties of the state vector, we propose a novel approach to transform the system dynamics into new forms such that the control input matrices of the transformed dynamics are invertible. This property plays an important role in designing control algorithms for UMSs. As a result, the concepts in [19], [20], [46], and [30] can be applied to stabilize the ballbot system by using the transformed dynamics.

- To enhance the robustness of the CHSMC, we propose new adaptive laws to handle model uncertainties and exogenous disturbance. With this enhancement, the requirement of the prior bounded values of the exogenous disturbance can be relaxed, and the chattering can be eliminated.

- By considering the applicability of the proposed controller in practice, the auxiliary system in [36], which is proposed for fictitious control inputs, is redesigned to reduce the input saturation effects of real actuators. In addition, the asymptotic convergence of auxiliary signals is proved rigorously.

The rest of this article is organized as follows. Section 2 describes the system model of the FNBD and the dynamic transformation. Section 3 presents the derivation of the proposed adaptive HSMC (AHSMC) with adaptive laws and the auxiliary system. Section 4 evaluates the effectiveness and robustness of our approach via comparative simulations. Finally, section 5 provides our conclusions.

Notations: $\|\bullet\|$ and $\|\bullet\|_{1}$ denotes the $l_{2}$-norm and $l_{1}$-norm of the vector $(\bullet)$, respectively. $|\bullet|$ is the absolute 
value of each element in the function, vector or matrix $(\bullet) \cdot \operatorname{diag}(\bullet)$ denotes the diagonal matrix constructed by the vector $(\bullet) . \sigma_{\max }(\bullet)$ denotes the maximum eigenvalue of the matrix $(\bullet) . \mathbf{I}_{3}=\operatorname{diag}(1,1,1)$.

\section{SYSTEM MODEL}

\subsection{Full nonlinear ballbot model}

In this section, the system model of the ballbot is presented. We use a ballbot model with the mechanical structure described in [4]. In the driving mechanism, three omnidirectional wheels are evenly assembled around the center of the ball with the zenith angle $45^{\circ}$. The coordinate system of an FNBD is shown in Figure 1. Here, $\varphi, \theta$, and $\psi$ represents the roll, pitch, and yaw angles, respectively. The system parameters are given in Table 1.

By using the Lagrange approach, the equations of motion can be represented in matrix form as follows [3]:

$$
\mathbf{M}(\mathbf{q}) \ddot{\mathbf{q}}+\mathbf{C}(\mathbf{q}, \dot{\mathbf{q}}) \dot{\mathbf{q}}+\mathbf{g}(\mathbf{q})=\mathbf{B}(\mathbf{q}) \tau-\mathbf{v}(\dot{\mathbf{q}}),
$$

where $\mathbf{q}=\left[\begin{array}{lllll}p_{x} & p_{y} & \theta & \varphi\end{array}\right]^{T}$ is the generalized coordinate vector, $\mathbf{M} \in \mathfrak{R}^{5 \times 5}$ is the inertia matrix, $\mathbf{C} \in \mathfrak{R}^{5 \times 5}$ is the Coriolis-centrifugal matrix, $\mathbf{g} \in \mathfrak{R}^{5}$ is the gravity vector, $\mathbf{B} \in \mathfrak{R}^{5 \times 3}$ is the input matrix, and $\boldsymbol{\tau}=\left[\begin{array}{lll}\tau_{1} & \tau_{2} & \tau_{3}\end{array}\right]^{T}$ is the input vector. These nominal system matrices can be represented as:

$$
\mathbf{M}=\left[\begin{array}{lllll}
m_{1} & m_{2} & m_{3} & m_{4} & m_{5} \\
m_{2} & m_{6} & m_{7} & m_{8} & m_{9} \\
m_{3} & m_{7} & m_{10} & 0 & m_{11} \\
m_{4} & m_{8} & 0 & m_{12} & m_{13} \\
m_{5} & m_{9} & m_{11} & m_{13} & m_{14}
\end{array}\right], \mathbf{C}=\left[\begin{array}{lllll}
c_{11} & c_{12} & c_{13} & c_{14} & c_{15} \\
c_{21} & c_{22} & c_{23} & c_{24} & c_{25} \\
c_{31} & c_{32} & c_{33} & c_{34} & c_{35} \\
c_{41} & c_{42} & c_{43} & 0 & c_{45} \\
c_{51} & c_{52} & c_{53} & c_{54} & c_{55}
\end{array}\right], \mathbf{B}=\frac{\sqrt{2}}{2} \frac{r_{s}}{r_{w}}\left[\begin{array}{llll}
b_{11} & b_{12} & b_{13} \\
b_{21} & b_{22} & b_{23} \\
b_{31} & b_{32} & b_{33} \\
b_{41} & b_{42} & b_{43} \\
b_{51} & b_{52} & b_{53}
\end{array}\right], \mathbf{g}=\left[\begin{array}{lllll}
0 & 0 & g_{3} & g_{4} & 0
\end{array}\right]^{T},
$$

where the elements of $\mathbf{M}, \mathbf{C}$, and $\mathbf{g}$ matrices are given in Appendix A. $\mathbf{v}(\dot{\mathbf{q}}) \in \mathfrak{R}^{5}$ is the vector of Coulomb and viscous frictions, it is defined as

$$
\mathbf{v}(\dot{\mathbf{q}})=\left[\varsigma_{p}^{d} \dot{p}_{x}+\varsigma_{p}^{s} \operatorname{sgn}\left(\dot{p}_{x}\right) \quad \varsigma_{p}^{d} \dot{p}_{y}+\varsigma_{p}^{s} \operatorname{sgn}\left(\dot{p}_{y}\right) \quad \varsigma_{b}^{d} \dot{\theta}+\varsigma_{b}^{s} \operatorname{sgn}(\dot{\theta}) \quad \varsigma_{b}^{d} \dot{\varphi}+\varsigma_{b}^{s} \operatorname{sgn}(\dot{\varphi}) \quad \varsigma_{y}^{d} \dot{\psi}+\varsigma_{y}^{s} \operatorname{sgn}(\dot{\psi})\right]^{T} .
$$

Here, $\varsigma_{p}^{d}, \varsigma_{p}^{s}, \varsigma_{b}^{d}, \varsigma_{b}^{s}, \varsigma_{y}^{d}$, and $\varsigma_{y}^{s}$ are friction coefficients. In practice, the friction effects are time-variant depending on elastic coefficients and characteristics of the ball and ground surfaces. Therefore, in this study, we consider the friction vector $\mathbf{v}(\dot{\mathbf{q}})$ as an element of exogenous disturbance.

Remark 1. The FNBD (1) is a MIMO, UMS, where the control input matrix B is noninvertible. Thus, conventional SMC, feedback linearization control, and HSMC algorithms cannot be applied directly to stabilize the system. In the next section, a dynamic transformation is proposed to tackle this problem.

\subsection{Dynamic transformation}

In this section, the FNBD is transformed into two subsystems such that the HSMC scheme can be applied to stabilize the whole system. The original generalized coordinate vector is transformed into two sub-vectors, i.e., $\mathbf{q}_{0}=\left[\begin{array}{lll}\theta & \varphi & \psi\end{array}\right]^{T}$ and $\mathbf{q}_{1}=\left[\begin{array}{lll}p_{x} & p_{y} \psi\end{array}\right]^{T}$. Based on these vectors, we derive two subsystems that are the orientation subsystem and the pose subsystem. Consider the effects of model uncertainties and exogenous disturbance, the equations of motion (1) can be transformed as

$$
\mathbf{M}_{i} \ddot{\mathbf{q}}_{i}+\mathbf{f}_{i}=\mathbf{B}_{i} \boldsymbol{\tau}+\Delta_{i}+\boldsymbol{\tau}_{m i}, i=0,1 .
$$

where $\boldsymbol{\tau}_{m i}$ stands for exogenous disturbance including the friction effects and $\boldsymbol{\Delta}_{i}$ is the vector of the lumped model uncertainties. Let $\breve{\mathbf{M}}_{i}$ and $\breve{\mathbf{f}}_{i}$ be the perturbation of the nominal matrices $\mathbf{M}_{i}$ and $\mathbf{f}_{i}, \boldsymbol{\Delta}_{i}$ is defined as: 


$$
\boldsymbol{\Delta}_{i}=-\breve{\mathbf{M}}_{i} \ddot{\mathbf{q}}_{i}-\breve{\mathbf{f}}_{i}, i=0,1 .
$$

this vector satisfies the following constraint [47]:

$$
\left|\boldsymbol{\Delta}_{i}\right|<\boldsymbol{\alpha}_{i 0}+\boldsymbol{\alpha}_{i 1}\|\mathbf{q}\|+\boldsymbol{\alpha}_{i 2}\|\dot{\mathbf{q}}\|^{2}
$$

where $\boldsymbol{\alpha}_{i 0}, \boldsymbol{\alpha}_{i 1}, \boldsymbol{\alpha}_{i 2}, \in \mathfrak{R}^{3}$ are positive constant vectors.

The transformed matrices can be obtained as

$$
\begin{aligned}
& \mathbf{M}_{0}=\mathbf{M}_{b 1}-\mathbf{M}_{b p 1} \mathbf{M}_{p 1}^{-1} \mathbf{M}_{p b 1}, \mathbf{M}_{1}=\mathbf{M}_{p 2}-\mathbf{M}_{p b 2} \mathbf{M}_{b 2}^{-1} \mathbf{M}_{b p 2}, \\
& \mathbf{B}_{0}=\mathbf{B}_{b 1}-\mathbf{M}_{b p 1} \mathbf{M}_{p 1}^{-1} \mathbf{B}_{p 1}, \mathbf{B}_{1}=\mathbf{B}_{p 2}-\mathbf{M}_{p b 2} \mathbf{M}_{b 2}^{-1} \mathbf{B}_{b 2}, \\
& \mathbf{f}_{0}=\mathbf{f}_{b 1}-\mathbf{M}_{b p 1} \mathbf{M}_{p 1}^{-1} \mathbf{f}_{p 1}, \mathbf{f}_{1}=\mathbf{f}_{p 1}-\mathbf{M}_{p b 1} \mathbf{M}_{b 1}^{-1} \mathbf{f}_{b 1}, \\
& \mathbf{f}_{b i}=\mathbf{C}_{b p i} \dot{\mathbf{q}}_{p}+\mathbf{C}_{b i} \dot{\mathbf{q}}_{b}+\mathbf{g}_{b i}, \mathbf{f}_{p i}=\mathbf{C}_{p i} \dot{\mathbf{q}}_{p}+\mathbf{C}_{p b i} \dot{\mathbf{q}}_{b}+\mathbf{g}_{p i}, \\
& \mathbf{M}_{b 1}=\left[\begin{array}{ccc}
m_{10} & 0 & m_{11} \\
0 & m_{12} & m_{13} \\
m_{11} & m_{13} & m_{14}
\end{array}\right], \mathbf{M}_{p 2}=\left[\begin{array}{lll}
m_{1} & m_{2} & m_{5} \\
m_{2} & m_{6} & m_{9} \\
m_{5} & m_{9} & m_{14}
\end{array}\right], \mathbf{M}_{p b 1}=\mathbf{M}_{b p 1}^{T}=\left[\begin{array}{lll}
m_{3} & m_{4} & m_{5} \\
m_{7} & m_{8} & m_{9}
\end{array}\right], \mathbf{M}_{p 1}=\left[\begin{array}{ll}
m_{1} & m_{2} \\
m_{2} & m_{6}
\end{array}\right], \\
& \mathbf{M}_{b p 2}=\mathbf{M}_{p b 2}^{T}=\left[\begin{array}{lll}
m_{3} & m_{7} & m_{11} \\
m_{4} & m_{8} & m_{13}
\end{array}\right], \mathbf{M}_{b 2}=\left[\begin{array}{cc}
m_{10} & 0 \\
0 & m_{12}
\end{array}\right] \text {, } \\
& \mathbf{C}_{b 1}=\left[\begin{array}{ccc}
c_{33} & c_{34} & c_{35} \\
c_{43} & 0 & c_{45} \\
c_{53} & c_{54} & c_{55}
\end{array}\right], \mathbf{C}_{p 2}=\left[\begin{array}{lll}
c_{11} & c_{12} & c_{15} \\
c_{21} & c_{22} & c_{25} \\
c_{51} & c_{52} & c_{55}
\end{array}\right], \mathbf{C}_{p 1}=\left[\begin{array}{ll}
c_{11} & c_{12} \\
c_{21} & c_{22}
\end{array}\right], \mathbf{C}_{b 2}=\left[\begin{array}{ll}
c_{33} & c_{34} \\
c_{43} & c_{44}
\end{array}\right], \\
& \mathbf{C}_{p b 1}=\left[\begin{array}{lll}
c_{13} & c_{14} & c_{15} \\
c_{23} & c_{24} & c_{25}
\end{array}\right], \mathbf{C}_{b p 1}=\left[\begin{array}{lll}
c_{31} & c_{41} & c_{51} \\
c_{32} & c_{42} & c_{52}
\end{array}\right]^{T}, \mathbf{C}_{b p 2}=\left[\begin{array}{lll}
c_{13} & c_{23} & c_{53} \\
c_{14} & c_{24} & c_{54}
\end{array}\right], \mathbf{C}_{p b 2}=\left[\begin{array}{lll}
c_{31} & c_{32} & c_{35} \\
c_{41} & c_{42} & c_{45}
\end{array}\right]^{T}, \\
& \mathbf{g}_{p 1}=\mathbf{0}^{2}, \mathbf{g}_{p 2}=\mathbf{0}^{3}, \mathbf{g}_{b 1}=\left[\begin{array}{ll}
\mathbf{g}_{b 2}^{T} & 0
\end{array}\right]^{T}, \mathbf{g}_{b 2}=\left[\begin{array}{ll}
g_{3} & g_{4}
\end{array}\right]^{T}, \\
& \mathbf{B}_{b 1}=\left[\begin{array}{lll}
b_{31} & b_{32} & b_{33} \\
b_{41} & b_{42} & b_{43} \\
b_{51} & b_{52} & b_{53}
\end{array}\right], \mathbf{B}_{p 2}=\left[\begin{array}{lll}
b_{11} & b_{12} & b_{13} \\
b_{21} & b_{22} & b_{23} \\
b_{51} & b_{52} & b_{53}
\end{array}\right], \mathbf{B}_{p 1}=\left[\begin{array}{lll}
b_{11} & b_{12} & b_{13} \\
b_{21} & b_{22} & b_{23}
\end{array}\right], \mathbf{B}_{b 2}=\left[\begin{array}{lll}
b_{31} & b_{32} & b_{33} \\
b_{41} & b_{42} & b_{43}
\end{array}\right] \text {. }
\end{aligned}
$$

By applying this transformation, the control input matrices $\mathbf{B}_{0}$ and $\mathbf{B}_{1}$ are invertible. The proof of this property is given in Appendix B. The next section presents the proposed adaptive controllers based on the HSMC scheme to stabilize the ballbot system.

\section{CONTROL SYSTEM DESIGN}

Let $\xi_{01}=\left[\theta \varphi \psi-\psi_{r}\right]^{T}$ and $\xi_{11}=\left[\begin{array}{lll}p_{x}-x_{r} & p_{y}-y_{r} \psi-\psi_{r}\end{array}\right]^{T}$ be the tracking errors of the two subsystems, where $\psi_{r}, x_{r}$, and $y_{r}$ are the set point of yaw angle and the positions along the $x$ - and $y$-axes, respectively. The control objective is to move the whole system to a given pose while simultaneously maintaining the system balance. The following assumptions are made.

Assumption 1. The reference signals and its time-derivative up to the second-order are bounded.

Assumption 2. During the operation of the ballbot, the body lean angles are constrained as

$$
|\varphi|,|\theta|<\Theta<\pi / 2 \text {. }
$$

Here, $\Theta$ is the largest sway angle from the upright posture of the body that the proposed controller can stabilize the ballbot system. Its magnitude depends on the power of the driving mechanism. The existing control approaches for ballbot are derived using linearized and decoupled models [1]-[3], where the 
small-angle approximation assumption is made for the dynamical modelling. However, the model uncertainty will increase at large body-lean angles due to the increase of the coupling effects and the inaccuracy of the linearized models. These effects can be reduced or avoided by using the full nonlinear dynamics.

Assumption 3. Three input actuations (motors) have the same constraint as follows.

$$
\left|\tau_{k}\right| \leq \tau_{b}, k=1,2,3
$$

where $\tau_{b}$ is a known bound of the motor torque. This value depends on the characteristics of motors.

Assumption 4. The difference between the saturated control input and the control input to be designed, $\breve{\boldsymbol{\tau}}=\left[\begin{array}{lll}\breve{\tau}_{1} & \breve{\tau}_{2} & \breve{\tau}_{3}\end{array}\right]^{T}$, is bounded, i.e.,

$$
\|\bar{\tau}\|=\left\|\tau_{s}-\boldsymbol{\tau}\right\| \leq \bar{\tau}_{\Delta},
$$

where $\bar{\tau}_{\Delta}$ is an unknown positive constant. This value should not be too big to guarantee the stability of the system [48].

Assumption 5. The matched uncertainty affecting to each transformed system $\boldsymbol{\tau}_{m i}$ is bounded.

Lemma 1. For any $\varepsilon>0$ and $\chi \in \mathfrak{R}^{n}$, the following inequality holds for an hyperbolic tangent function.

$$
\chi^{T} \tanh (\chi / \varepsilon) \geq 0
$$

The proof of this property is given in Appendix C.

\subsection{Adaptive hierarchical sliding mode control}

In previous studies [1], [4], [5], control algorithms are separately proposed for the decoupled dynamics of ballbots. Thus, each decoupled dynamics is stabilized by one controller. In contrast, in this paper, thanks to the transformed dynamics (3), a unique controller can be proposed to stabilize the whole nonlinear system.

The system (3) is rewritten in the tracking form as

$$
\left\{\begin{array}{l}
\dot{\xi}_{i 1}=\boldsymbol{\xi}_{i 2} \\
\dot{\xi}_{i 2}=\mathbf{h}_{i}+\mathbf{M}_{i}^{-1} \mathbf{B}_{i} \boldsymbol{\tau}_{s}+\mathbf{M}_{i}^{-1} \boldsymbol{\Delta}_{i}+\mathbf{M}_{i}^{-1} \mathbf{B}_{i} \boldsymbol{\tau}_{m i}
\end{array},\right.
$$

where $\mathbf{h}_{i}=-\mathbf{M}_{i}^{-1} \mathbf{f}_{i}-\dot{\boldsymbol{\xi}}_{i 2 r}, i=0,1, \boldsymbol{\xi}_{02 r}=\left[\begin{array}{lll}0 & 0 & \dot{\psi}_{r}\end{array}\right]^{T}, \boldsymbol{\xi}_{12 r}=\left[\begin{array}{lll}\dot{x}_{r} & \dot{y}_{r} \dot{\psi}_{r}\end{array}\right]^{T}$, and $\boldsymbol{\tau}_{s}=\left[\begin{array}{lll}\tau_{s 1} & \tau_{s 2} & \tau_{s 3}\end{array}\right]^{T}$ is the saturated control input, which is defined as follows.

$$
\tau_{s k}=\left\{\begin{array}{ll}
\tau_{b} \operatorname{sgn}\left(u_{k}\right), & \left|\tau_{k}\right|>\tau_{b} \\
\tau_{k}, & \left|\tau_{k}\right| \leq \tau_{b}
\end{array}, k=1,2,3 .\right.
$$

The first level of the hierarchy is defined by the two sliding surfaces of the two subsystems that are the orientation subsystem $\mathbf{s}_{0}=\left[\begin{array}{lll}s_{\theta} & s_{\varphi} & s_{\psi}\end{array}\right]^{T}$ and the pose subsystem $\mathbf{s}_{1}=\left[\begin{array}{lll}s_{x} & s_{y} & s_{\psi}\end{array}\right]^{T}$. These subsystems are defined as

$$
\mathbf{s}_{i}=\Upsilon_{i 0} \xi_{i 2}+\Upsilon_{i 1} \xi_{i 1}
$$

where $\Upsilon_{i 0}, \Upsilon_{i 1} \in \mathfrak{R}^{3 \times 3}, i=0,1$, are diagonal, positive definite matrices.

Using the HSMC scheme, the second sliding surface is constructed by aggregating the sliding surfaces from the first-level [18] [42] as follows.

$$
\mathbf{s}_{\Sigma}=\sum_{i=0,1} \overline{\boldsymbol{\Gamma}}_{i} \mathbf{s}_{i}
$$

Here, $\overline{\boldsymbol{\Gamma}}_{i}=\operatorname{diag}\left(\bar{\Gamma}_{i 0}, \bar{\Gamma}_{i 1}, \bar{\Gamma}_{i 2}\right)$ is a weighting matrix.

The equivalent control input for each subsystem can be obtained as

$$
\boldsymbol{\tau}_{e q i}=-\left(\Upsilon_{i 0} \mathbf{M}_{i}^{-1} \mathbf{B}_{i}\right)^{-1}\left(\Upsilon_{i 0} \mathbf{h}_{i}+\Upsilon_{i 1} \xi_{i 2}\right), i=0,1 .
$$


The CHSMC control law is determined as [18]

$$
\boldsymbol{\tau}_{c}=\boldsymbol{\tau}_{s w}+\sum_{i=0,1} \boldsymbol{\tau}_{e q i},
$$

where $\boldsymbol{\tau}_{s w}$ is the switching law, it is determined as

$$
\boldsymbol{\tau}_{s w}=-\left(\sum_{i=0,1} \Pi_{i}\right)^{-1}\left[\boldsymbol{\Xi}_{1} \operatorname{sgn}\left(\mathbf{s}_{\Sigma}\right)+\mathbf{\Xi}_{2} \mathbf{s}_{\Sigma}+\sum_{i=0,1} \Pi_{i} \boldsymbol{\tau}_{e q 1-i}\right]
$$

where $\boldsymbol{\Xi}_{i} \in \mathfrak{R}^{3 \times 3}$ is a diagonal positive definite matrix and $\Pi_{i}=\overline{\boldsymbol{\Gamma}}_{i} \Upsilon_{i 0} \mathbf{M}_{i}^{-1} \mathbf{B}_{i}$.

To enhance the robustness of the CHSMC, the following adaptive HSMC law is proposed as

$$
\boldsymbol{\tau}=\boldsymbol{\tau}_{c}+\boldsymbol{\tau}_{a d},
$$

where the adaptive law $\boldsymbol{\tau}_{a d}$ is proposed as follows:

$$
\boldsymbol{\tau}_{a d}=-\left(\left\|\mathbf{S}_{\Sigma}\right\|^{2}+\varepsilon\right)^{-1}\left(\sum_{i=0,1} \Pi_{i}\right)^{-1} \mathbf{s}_{\Sigma}\left|\mathbf{s}_{\Sigma}^{T}\right|\left\{\sum_{i=0,1}\left|\Pi_{i} \mathbf{B}_{i}^{-1}\right|\left(\hat{\boldsymbol{\alpha}}_{i 0}+\hat{\boldsymbol{\alpha}}_{i 1}\|\mathbf{q}\|+\hat{\boldsymbol{\alpha}}_{i 2}\|\dot{\mathbf{q}}\|^{2}\right)\right\}
$$

Here, $\hat{\boldsymbol{\alpha}}_{i k} \in \mathfrak{R}^{3}, k=0,1,2$, is the estimated vector of $\boldsymbol{\alpha}_{i k}$ and is proposed as

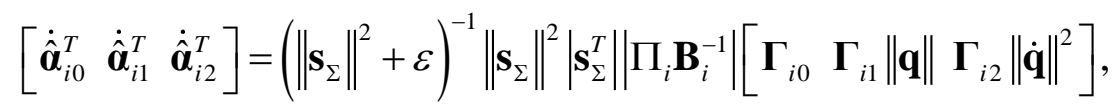

where $\Gamma_{i k} \in \mathfrak{R}^{3 \times 3}$ is a diagonal positive definite matrix and $\varepsilon$ is a small positive constant.

Remark 2. The parameter $\varepsilon$ specifies the boundary layer width and its roles are to (i) avoid the singularity of $\boldsymbol{\tau}_{a d}$ in (17) when $\left\|\mathbf{S}_{\Sigma}\right\|=0$ and (ii) avoid the continuous increase of the adaptive terms in (19) when $\left\|\mathbf{s}_{\Sigma}\right\|$ closes to zero, i.e., the system states locate in the vicinity of the origin. However, $\varepsilon$ should not be too small to avoid the chattering phenomenon of $\tau_{a d}$ when $\left\|\mathbf{s}_{\Sigma}\right\|$ approaches to the vicinity of the origin.

Remark 3. By applying the HSMC, the asymptotic convergence of $\mathbf{s}_{\Sigma}$ can be obtained. From (10)-(13), we can observe that both the system states and the tracking vectors of the subsystems are constructed such that the first and second rows of $\mathbf{s}_{\Sigma}$ represent the frontal and sagittal dynamic model as per [4], [5]. Also, the third row contains only the tracking error of the horizontal dynamics. The second sliding surface (13) can be rewritten as

$$
\mathbf{s}_{\Sigma}=\left[\begin{array}{l}
\bar{\Gamma}_{00} s_{\theta}+\bar{\Gamma}_{10} s_{x} \\
\bar{\Gamma}_{01} s_{\varphi}+\bar{\Gamma}_{11} s_{y} \\
\bar{\Gamma}_{02} s_{\psi}+\bar{\Gamma}_{12} s_{\psi}
\end{array}\right],
$$

Here, the third row of $\mathbf{s}_{\Sigma}$ (20) only contains the tracking error of $s_{\psi}$, i.e., the heading motion. Thus, we can select $\bar{\Gamma}_{02}, \bar{\Gamma}_{12}>0$. Moreover, by considering the first and second rows of (20) and using Lemma 2 in [42], the rest elements of $\overline{\boldsymbol{\Gamma}}_{i}$ are tuned under the following constraints:

$$
\bar{\Gamma}_{00}, \bar{\Gamma}_{01}, \bar{\Gamma}_{10}>0 \text { and } \bar{\Gamma}_{11}<0 \text {. }
$$

Lemma 2. If the sliding surface $\mathbf{s}_{\Sigma}$ converges to zero as $t \rightarrow \infty$, then the asymptotic convergence of the tracking error vectors can be achieved. As a result, the operation of the whole system can be governed by one controller.

The proof of Lemma is provided in Appendix D.

Theorem 1. Consider the FNBD under the tracking form (10). If the control input is proposed as (17), with the adaptive laws are determined as (18) and (19), and the sliding surfaces are defined as (12) and (13), then the sliding surfaces $\mathbf{s}_{\Sigma}, \mathbf{s}_{0}$, and $\mathbf{s}_{1}$ asymptotically converge to zero. As a result, all the tracking error vectors 
of the system can be stabilized, and the control objective of the system can be obtained.

Proof. A Lyapunov function candidate is defined as

$$
V_{\Sigma}=(1 / 2) \mathbf{s}_{\Sigma}^{T} \mathbf{s}_{\Sigma}+(1 / 2) \sum_{i=0,1} \sum_{k=0,1,2} \tilde{\boldsymbol{\alpha}}_{i k}^{T} \boldsymbol{\Gamma}_{i k}^{-1} \tilde{\boldsymbol{\alpha}}_{i k}
$$

where $\tilde{\boldsymbol{\alpha}}_{i j}=\boldsymbol{\alpha}_{i j}-\hat{\boldsymbol{\alpha}}_{i j}$.

Differentiating (22) with respect to time yields

$$
\dot{V}_{\Sigma}=\mathbf{s}_{\Sigma}^{T} \dot{\mathbf{s}}_{\Sigma}-\sum_{i=0,1} \sum_{k=0,1,2} \dot{\hat{\boldsymbol{\alpha}}}_{i k}^{T} \boldsymbol{\Gamma}_{i k}^{-1} \tilde{\boldsymbol{\alpha}}_{i k},
$$

where,

$$
\dot{\mathbf{S}}_{\Sigma}=\sum_{i} \overline{\boldsymbol{\Gamma}}_{i}\left(\Upsilon_{i 0}\left(\mathbf{h}_{i}+\mathbf{M}_{i}^{-1} \mathbf{B}_{i}(\boldsymbol{\tau}+\overline{\boldsymbol{\tau}})+\mathbf{M}_{i}^{-1} \boldsymbol{\Delta}_{i}+\mathbf{M}_{i}^{-1} \mathbf{B}_{i} \boldsymbol{\tau}_{m i}\right)+\Upsilon_{i 1} \xi_{i 2}\right)
$$

Substituting the control laws (17) into (23) yields

$$
\dot{V}_{\Sigma}=-\mathbf{s}_{\Sigma}^{T} \boldsymbol{\Xi}_{1} \operatorname{sgn}\left(\mathbf{s}_{\Sigma}\right)-\mathbf{s}_{\Sigma}^{T} \boldsymbol{\Xi}_{2} \mathbf{s}_{\Sigma}-\sum_{i=0,1} \sum_{k=0,1,2} \dot{\hat{\boldsymbol{\alpha}}}_{i k}^{T} \boldsymbol{\Gamma}_{i k}^{-1} \tilde{\boldsymbol{\alpha}}_{i k}+\mathbf{s}_{\Sigma}^{T} \sum_{i=0,1} \Pi_{i}\left(\breve{\boldsymbol{\tau}}+\boldsymbol{\tau}_{m i}+\mathbf{B}_{i}^{-1} \boldsymbol{\Delta}_{i}\right)+\mathbf{s}_{\Sigma}^{T} \sum_{i=0,1} \Pi_{i} \boldsymbol{\tau}_{a d} .
$$

Using (18) and (25), we obtain

$$
\begin{aligned}
\dot{V}_{\Sigma} \leq & -\mathbf{s}_{\Sigma}^{T} \boldsymbol{\Xi}_{1} \operatorname{sgn}\left(\mathbf{s}_{\Sigma}\right)-\mathbf{s}_{\Sigma}^{T} \boldsymbol{\Xi}_{2} \mathbf{s}_{\Sigma}-\sum_{i=0,1} \sum_{k=0,1,2} \dot{\hat{\boldsymbol{\alpha}}}_{i k}^{T} \boldsymbol{\Gamma}_{i k}^{-1} \tilde{\boldsymbol{\alpha}}_{i k}+\mathbf{s}_{\Sigma}^{T} \sum_{i=0,1} \Pi_{i}\left(\breve{\boldsymbol{\tau}}+\boldsymbol{\tau}_{m i}\right) \\
& +\left(\left\|\mathbf{S}_{\Sigma}\right\|^{2}+\varepsilon\right)^{-1}\left\|\mathbf{s}_{\Sigma}\right\|^{2}\left|\mathbf{s}_{\Sigma}^{T}\right|\left\{\sum_{i=0,1}\left|\Pi_{i} \mathbf{B}_{i}^{-1}\right|\left(\left|\boldsymbol{\Delta}_{i}\right|-\hat{\boldsymbol{\alpha}}_{i 0}-\hat{\boldsymbol{\alpha}}_{i 1}\|\mathbf{q}\|-\hat{\boldsymbol{\alpha}}_{i 2}\|\dot{\mathbf{q}}\|^{2}\right)\right\}+\bar{\varepsilon} \mathbf{s}_{\Sigma}^{T} \sum_{i=0,1} \Pi_{i}\left(\mathbf{B}_{i}^{-1} \boldsymbol{\Delta}_{i}\right) .
\end{aligned}
$$

where $\bar{\varepsilon}=\left(\left\|\mathbf{S}_{\Sigma}\right\|^{2}+\varepsilon\right)^{-1} \varepsilon$.

Substituting the adaptive laws (19) into (26) yields

$$
\begin{aligned}
\dot{V}_{\Sigma} \leq & -\mathbf{s}_{\Sigma}^{T} \boldsymbol{\Xi}_{1} \operatorname{sgn}\left(\mathbf{s}_{\Sigma}\right)-\mathbf{s}_{\Sigma}^{T} \boldsymbol{\Xi}_{2} \mathbf{s}_{\Sigma}+\mathbf{s}_{\Sigma}^{T} \sum_{i=0,1} \Pi_{i}\left(\breve{\boldsymbol{\tau}}+\boldsymbol{\tau}_{m i}\right)+\bar{\varepsilon} \mathbf{s}_{\Sigma}^{T} \sum_{i=0,1} \Pi_{i}\left(\mathbf{B}_{i}^{-1} \boldsymbol{\Delta}_{i}\right) \\
& +\left(\left\|\mathbf{S}_{\Sigma}\right\|^{2}+\varepsilon\right)^{-1}\left\|\mathbf{s}_{\Sigma}\right\|^{2}\left|\mathbf{s}_{\Sigma}^{T}\right|\left\{\sum_{i=0,1}\left|\Pi_{i} \mathbf{B}_{i}^{-1}\right|\left(\left|\boldsymbol{\Delta}_{i}\right|-\boldsymbol{\alpha}_{i 0}-\boldsymbol{\alpha}_{i 1}\|\mathbf{q}\|-\boldsymbol{\alpha}_{i 2}\|\dot{\mathbf{q}}\|^{2}\right)\right\} .
\end{aligned}
$$

From (5) and (27) we obtain

$$
\dot{V}_{\Sigma} \leq-\vartheta_{1}\left\|\mathbf{s}_{\Sigma}\right\|_{1}-\mathbf{s}_{\Sigma}^{T} \Xi_{2} \mathbf{s}_{\Sigma}
$$

where $\vartheta_{1}=\sigma_{\text {min }}\left(\boldsymbol{\Xi}_{1}\right)-\left\|\sum_{i=0,1} \Pi_{i}\left(\breve{\boldsymbol{\tau}}+\boldsymbol{\tau}_{m i}+\bar{\varepsilon} \mathbf{B}_{i}^{-1} \boldsymbol{\Delta}_{i}\right)\right\|_{1}$.

If the control gains of the matrix $\boldsymbol{\Xi}_{1}$ are tuned such that

$$
\vartheta_{1} \geq 0
$$

then we obtain

$$
\dot{V}_{\Sigma} \leq 0
$$

Integrating both sides of (28) yields

$$
V_{\Sigma}(t)-V_{\Sigma}(0) \leq \int_{0}^{t}-\left[\vartheta_{1}\left\|\mathbf{s}_{\Sigma}\right\|_{1}+\sigma_{\max }\left(\boldsymbol{\Xi}_{2}\right)\left\|\mathbf{s}_{\Sigma}\right\|^{2}\right] d \tau .
$$

The term $V_{\Sigma}(0)$ can be rewritten as

$$
V_{\Sigma}(0) \geq V_{\Sigma}(t)+\int_{0}^{t}\left[\vartheta_{1}\left\|\mathbf{s}_{\Sigma}\right\|_{1}+\sigma_{\max }\left(\mathbf{\Xi}_{2}\right)\left\|\mathbf{s}_{\Sigma}\right\|^{2}\right] d \tau \geq \int_{0}^{t}\left[\vartheta_{1}\left\|\mathbf{s}_{\Sigma}\right\|_{1}+\sigma_{\max }\left(\boldsymbol{\Xi}_{2}\right)\left\|\mathbf{s}_{\Sigma}\right\|^{2}\right] d \tau,
$$

thereby yielding

$$
\lim _{t \rightarrow \infty} \int_{0}^{t}\left[\vartheta_{1}\left\|\mathbf{S}_{\Sigma}\right\|_{1}+\sigma_{\max }\left(\boldsymbol{\Xi}_{2}\right)\left\|\mathbf{s}_{\Sigma}\right\|^{2}\right] d \tau \leq V_{\Sigma}(0)<\infty
$$

which implies 


$$
\mathbf{s}_{\Sigma} \in l_{2}
$$

Using (22) and (32) we obtain

$$
V_{\Sigma}(t) \leq V_{\Sigma}(0)+\int_{0}^{t}\left[\vartheta_{1}\left\|\mathbf{s}_{\Sigma}\right\|_{1}+\sigma_{\max }\left(\boldsymbol{\Xi}_{2}\right)\left\|\mathbf{s}_{\Sigma}\right\|^{2}\right] d \tau \leq \infty,
$$

which implies

$$
\mathbf{s}_{\Sigma} \in l_{\infty}
$$

Using (30) and (36) yields

$$
\dot{\mathbf{S}}_{\Sigma} \in l_{\infty}
$$

From (34) and (37), using Barbalat's lemma [49], we obtain $\mathbf{s}_{\Sigma} \rightarrow \mathbf{0}$ as $t \rightarrow \infty$. In addition, the asymptotic convergence of sliding surfaces $s_{0}$ and $s_{1}$ is also obtained based on Lemma 2 . Thus, the control objective of the system is achieved. This means that the whole system is transformed into the designated position and heading angle while maintaining its upright posture, i.e., the control objective is achieved. Q.E.D.

It is noted that despite possessing the robustness of SMC, the HSMC scheme only guarantees asymptotic convergence for the subsystem sliding surfaces of underactuated systems [18], [33]-[35], [37], [42].

Remark 4. Without considering the input saturation, the term $\breve{\tau}$ is considered as part of matched uncertainties. This term is bounded as Assumptions 4 and 5. Thus, the condition (29) can be achieved by trial-and-error method. In addition, the uncertain term $\bar{\varepsilon} \mathbf{B}_{i}^{-1} \boldsymbol{\Delta}_{i}$ in $\vartheta_{1}$ affects the system in the same channel with control input vector. Thus, $\bar{\varepsilon} \mathbf{B}_{i}^{-1} \boldsymbol{\Delta}_{i}$ can be considered as an element of the matched uncertainty vector $\boldsymbol{\tau}_{m i}$.

\subsection{Adaptive hierarchical sliding mode control under input saturation}

Motivated by [36], we design the following auxiliary system to examine the influence of input saturation for each motor.

$$
\dot{\boldsymbol{\mu}}=\left\{\begin{array}{ll}
\mathbf{0}, & \boldsymbol{\mu}=\mathbf{0} \\
\dot{\overline{\boldsymbol{\mu}}}=-\mathbf{K}_{\bar{\mu}} \boldsymbol{\mu}-\|\boldsymbol{\mu}\|_{2}^{-1} \boldsymbol{\mu}\left|\mathbf{s}_{\Sigma}^{T}\left(\sum_{i=0,1} \Pi_{i}\right) \check{\boldsymbol{\tau}}\right|, & \text { otherwise }
\end{array},\right.
$$

where $\mathbf{K}_{\bar{\mu}} \in \mathfrak{R}^{3 \times 3}$ is a diagonal positive definite matrix, $\boldsymbol{\mu}=\left[\begin{array}{lll}\mu_{1} & \mu_{2} & \mu_{3}\end{array}\right]^{T}$ and $\overline{\boldsymbol{\mu}}=\left[\begin{array}{lll}\bar{\mu}_{1} & \bar{\mu}_{2} & \bar{\mu}_{3}\end{array}\right]^{T}$. Then, each term $\dot{\boldsymbol{\mu}}$ is updated as:

$$
\dot{\mu}_{k}=\left\{\begin{array}{ll}
\dot{\bar{\mu}}_{k} & ,\left|\mu_{k}\right|>\varsigma_{\bar{\mu}} \\
\bar{\tau}_{k} & ,\left|\mu_{k}\right| \leq \varsigma_{\bar{\mu}}
\end{array}, k=1,2,3,\right.
$$

where $\varsigma_{\bar{\mu}}$ is a small positive constant.

Remark 5. The constant $\varsigma_{\bar{\mu}}$ acts as a boundary width for the update of the auxiliary signal $\mu_{k}$ in (39). It should not be too small to avoid large oscillations of $\dot{\overline{\boldsymbol{\mu}}}$ in (38) when $\|\boldsymbol{\mu}\|_{2}$ is close to the origin. If all terms of $\boldsymbol{\mu}$ equal to zero, then $\dot{\boldsymbol{\mu}}=\mathbf{0}$. Otherwise, each term of $\dot{\boldsymbol{\mu}}$ is updated as conditions in (38) and (39). The interested readers are encouraged to refer to Remarks 5 and 6 in [36] for more detail about how to design the auxiliary system (38).

To handle the effects of input saturation, chattering phenomenon, and exogenous disturbance, we proposed an AHSMC as

$$
\boldsymbol{\tau}=\boldsymbol{\tau}_{s w n}+\boldsymbol{\tau}_{a d}-\mathbf{K}_{\mu} \boldsymbol{\mu}+\sum_{i=0,1}\left(\boldsymbol{\tau}_{e q i}-\hat{\boldsymbol{\tau}}_{m i}\right)
$$


where $\mathbf{K}_{\mu} \in \mathfrak{R}^{3 \times 3}$ is a diagonal, positive definite matrix. Based on the auxiliary system (38)- (39), we can obtain $\operatorname{sgn}\left(\tau_{k}\right)=\operatorname{sgn}\left(\mu_{k}\right)$. Thus, $\mathbf{K}_{\mu}$ is tuned to reduce the input saturation effects. However, it should not be too large to avoid degrading control performance and causing instability of the system when an input saturation phenomenon occurs.

To avoid chattering phenomenon caused by the signum function, the switching law $\boldsymbol{\tau}_{s w n}$ is modified from (16) as

$$
\boldsymbol{\tau}_{s w n}=-\left(\sum_{i=0,1} \Pi_{i}\right)^{-1}\left[\boldsymbol{\Xi}_{1} \tanh \left(\varsigma_{t} \mathbf{s}_{\Sigma}\right)+\boldsymbol{\Xi}_{2} \mathbf{s}_{\Sigma}+\sum_{i=0,1} \Pi_{i} \boldsymbol{\tau}_{e q 1-i}\right]
$$

where $\varsigma_{t}$ is a positive constant, it is chosen to adjust the boundary layer around the sliding surface $\mathbf{s}_{\Sigma}$.

The adaptive law $\hat{\boldsymbol{\tau}}_{m i}$ is updated as:

$$
\dot{\hat{\boldsymbol{\tau}}}_{m i}=\boldsymbol{\Gamma}_{m i} \Pi_{i} \mathbf{s}_{\Sigma}
$$

Lemma 3. Using the proposed control algorithm, if $\widetilde{\boldsymbol{\tau}}=0$ and exists $\mu_{i} \neq 0$, then $\mu_{i}$ tends to be zero as $t \rightarrow \infty$.

Proof.

From (38)-(39), when there are no input saturation effects, i.e., $\breve{\boldsymbol{\tau}}=0$, the auxiliary vector $\boldsymbol{\mu}$ remains the following form

$$
\dot{\boldsymbol{\mu}}=-\mathbf{K}_{\bar{\mu}} \boldsymbol{\mu} .
$$

The following Lyapunov candidate is adopted

$$
V_{\tau}=\frac{1}{2} \boldsymbol{\mu}^{T} \mathbf{K}_{\bar{\mu}}^{-1} \boldsymbol{\mu}
$$

Differentiating (44) yields

$$
\dot{V}_{\tau}=-\boldsymbol{\mu}^{T} \boldsymbol{\mu}<0, \forall \boldsymbol{\mu} \neq \mathbf{0}
$$

It means that $\mu_{i}$ decays over time.

On the other hand, if the control input values grow higher than its upper bounds, then the ultimate control input $\boldsymbol{\tau}$ in (40) is decreased by an amount of $\mathbf{K}_{\mu} \boldsymbol{\mu}$, and vice versa. Q.E.D.

Theorem 2. Consider the FNBD under the tracking form (10). If the control input is proposed as (40), with the adaptive law determined as (18), (19), and (42). Also, the sliding surfaces are defined as (12) and (13), then the two sliding surfaces of the hierarchy asymptotically converge to zero and the desired control performance of the system is achieved. In addition, the input saturation and chattering problems can be reduced or avoided.

$$
V_{\bar{\Sigma}}=(1 / 2) \mathbf{S}_{\Sigma}^{T} \mathbf{S}_{\Sigma}+(1 / 2) \sum_{i=0,1} \sum_{k=0,1,2} \tilde{\boldsymbol{\alpha}}_{i k}^{T} \boldsymbol{\Gamma}_{i k}^{-1} \tilde{\boldsymbol{\alpha}}_{i k}+(1 / 2) \sum_{i=0,1} \tilde{\boldsymbol{\tau}}_{m i}^{T} \boldsymbol{\Gamma}_{m i}^{-1} \tilde{\boldsymbol{\tau}}_{m i}+(1 / 2) \boldsymbol{\mu}^{T} \boldsymbol{\mu}
$$

where $\tilde{\boldsymbol{\tau}}_{m i}=\boldsymbol{\tau}_{m i}-\hat{\boldsymbol{\tau}}_{m i}$.

Proof.

Differentiating (46), then using the proposed control law (40) and Remark 4, we obtain

$$
\dot{V}_{\bar{\Sigma}} \leq-\mathbf{s}_{\Sigma}^{T} \Xi_{1} \tanh \left(\varsigma_{t} \mathbf{s}_{\Sigma}\right)-\mathbf{s}_{\Sigma}^{T} \Xi_{2} \mathbf{s}_{\Sigma}+\mathbf{s}_{\Sigma}^{T} \sum_{i=0,1} \Pi_{i} \tilde{\boldsymbol{\tau}}_{m i}-\sum_{i=0,1} \tilde{\boldsymbol{\tau}}_{m i}^{T} \boldsymbol{\Gamma}_{m i}^{-1} \dot{\boldsymbol{\tau}}_{m i}+\mathbf{s}_{\Sigma}^{T} \sum_{i=0,1} \Pi_{i}\left(\breve{\boldsymbol{\tau}}-\mathbf{K}_{\mu} \boldsymbol{\mu}\right)+\boldsymbol{\mu}^{T} \dot{\boldsymbol{\mu}} .
$$

We have

$$
-\mathbf{S}_{\Sigma}^{T} \boldsymbol{\Xi}_{\mu} \boldsymbol{\mu} \leq(1 / 2) \mathbf{s}_{\Sigma}^{T} \mathbf{S}_{\Sigma}+(1 / 2)\left(\boldsymbol{\Xi}_{\mu} \boldsymbol{\mu}\right)^{T} \boldsymbol{\Xi}_{\mu} \boldsymbol{\mu}
$$

where $\boldsymbol{\Xi}_{\mu}=\left(\sum_{i} \Pi_{i}\right) \mathbf{K}_{\mu}$.

Using (42), (47) and (48) yields 


$$
\dot{V}_{\bar{\Sigma}} \leq-\mathbf{s}_{\Sigma}^{T} \Xi_{1} \tanh \left(\varsigma_{t} \mathbf{s}_{\Sigma}\right)-\mathbf{s}_{\Sigma}^{T} \vartheta_{2} \mathbf{s}_{\Sigma}+\boldsymbol{\mu}^{T} \dot{\boldsymbol{\mu}}+\left|\mathbf{s}_{\Sigma}^{T} \sum_{i=0,1} \Pi_{i} \tilde{\tau}\right|+(1 / 2)\left(\boldsymbol{\Xi}_{\mu} \boldsymbol{\mu}\right)^{T} \boldsymbol{\Xi}_{\mu} \boldsymbol{\mu},
$$

where $\vartheta_{2}=\Xi_{2}-(1 / 2) \mathbf{I}_{2}$.

Substituting (38) into (49) yields

$$
\dot{V}_{\bar{\Sigma}} \leq-\mathbf{s}_{\Sigma}^{T} \boldsymbol{\Xi}_{1} \tanh \left(\varsigma_{t} \mathbf{s}_{\Sigma}\right)-\mathbf{s}_{\Sigma}^{T} \vartheta_{2} \mathbf{s}_{\Sigma}-\boldsymbol{\mu}^{T}\left[\mathbf{K}_{\bar{\mu}}-(1 / 2) \mathbf{\Xi}_{\mu}^{T} \boldsymbol{\Xi}_{\mu}\right] \boldsymbol{\mu} .
$$

Using Lemma 1 and if the control gains in matrices are tuned such that

$$
\sigma_{\min }\left(\mathbf{K}_{\bar{\mu}}\right) \geq \sigma_{\max }^{2}\left(\boldsymbol{\Xi}_{\mu}\right) / 2 \text {, }
$$

then we have

$$
\dot{V}_{\bar{\Sigma}} \leq 0 .
$$

In the same manner, by invoking steps from (31) - (37), the asymptotic convergence of $\mathbf{s}_{\Sigma}$ can be obtained by Barbalat's Lemma. As a result, the tracking error vectors can be stabilized thanks to the results in Lemma 2. Thus, the control objective of the subsystems is achieved. Using the auxiliary system (38), the input saturation can be reduced or avoided by Lemma 3. Also, from (50) we can observe that the elements of the matrix $\boldsymbol{\Xi}_{1}$ can be chosen with arbitrary small-positive values to satisfy (52); therefore, the chattering can be eliminated. Q.E.D.

Remark 6. The auxiliary system (38) is used to compensate for the input saturation effects when $\breve{\boldsymbol{\tau}} \neq \mathbf{0}$ to make $\breve{\boldsymbol{\tau}}$ smaller and satisfy Assumption 4. This phenomenon happens at large values of tracking errors. If $\|\tilde{\boldsymbol{\tau}}\|$ is not too big as Assumption 4, then using the proposed algorithm (40), the tracking errors become smaller and reach their desired values. As a result, we obtain $\breve{\boldsymbol{\tau}}=\mathbf{0}$. Then, the convergence of auxiliary signals is obtained by Lemma 3 .

Remark 7. Since $\boldsymbol{\Xi}_{\mu}$ only contains elements from the system parameters, the condition (51) can be obtained by trial-and-error method. In addition, the condition (29), which relies on matched uncertainties, is relaxed under the proposed control law (40).

\section{SIMULATIONS AND DISCUSSIONS}

In this section, numerical simulations are conducted to highlight the control performance of the proposed algorithm, the AHSMC, and compare it with the CHSMC. The model parameters are shown in Table 1. Control parameters for the CHSMC are tuned and selected as in Table 2. These parameters are shared for both CHSMC and AHSMC. Then, the control gains for adaptive laws and the auxiliary system will be tuned for the AHSMC. The adaptive gains are selected as $\boldsymbol{\Gamma}_{0 \mathrm{k}}=0.01 \times \mathbf{I}_{3}, \boldsymbol{\Gamma}_{1 \mathrm{k}}=0.05 \times \mathbf{I}_{3}, \boldsymbol{\Gamma}_{m i}=1.2 \times \mathbf{I}_{3}$, and $\varepsilon=0.1$. The gains of the auxiliary system are chosen as $\mathbf{K}_{\mu}=2.8 \times \mathbf{I}_{3}$ and $\mathbf{K}_{\bar{\mu}}=100 \times \mathbf{I}_{3}$. The motor torque is bounded at $\tau_{b}=60 \mathrm{~N}$. The parameters $\varsigma_{t}=50$ and $\varsigma_{\bar{\mu}}=0.01$. We conduct simulations based on three general working scenarios that usually happen during the operation of a real ridable ballbot. In all scenarios, the nominal dynamics are assumed to be affected by the variations of system parameters and model uncertainties. Thus, the moment of inertia and body mass are assumed to be changed to $I_{b x}=21 \mathrm{~kg} \cdot \mathrm{m}^{2}, I_{b y}=19 \mathrm{~kg} \cdot \mathrm{m}^{2}, I_{b z}=1.5$ $\mathrm{kg} \cdot \mathrm{m}^{2}$, and $m_{s}=176 \mathrm{~kg}$, due to the change of a rider mass. In addition, the friction coefficients are selected as $\varsigma_{p}^{d}=2 \mathrm{~N} \cdot \mathrm{s} / \mathrm{m}, \varsigma_{p}^{s}=1 \mathrm{~N}, \varsigma_{b}^{s}=0.5 \mathrm{~N} \cdot \mathrm{m}, \varsigma_{b}^{d}=2 \mathrm{~N} \cdot \mathrm{m} \cdot \mathrm{s} / \mathrm{rad}, \varsigma_{y}^{s}=0.1 \mathrm{~N} \cdot \mathrm{m}$, and $\varsigma_{y}^{d}=1 \mathrm{~N} \cdot \mathrm{m} \cdot \mathrm{s} / \mathrm{rad}$. Three performance indexes (PI) are used for the comparative results that are the maximum absolute error (MAE), root mean square error of tracking error (RMSE) [36], and integration of absolute error (IAE). The IAE of a tracking error vector $\zeta$ is defined as

$$
\operatorname{IAE}(\zeta)=\sum_{k=0}^{T}|\zeta(k)|
$$


where $T$ is the total simulation time.

Remark 8. We can observe that the proposed AHSMC in (40) is an improved version of the CHSMC in (15) . Thus, the tuning process of the AHSMC includes two steps: (i) find a proper control set for the CHSMC such that the whole system can be stabilized by $\boldsymbol{\tau}_{c}$, (ii) select proper parameters for the adaptive terms of $\boldsymbol{\tau}_{a d}$ and the auxiliary system such that the proposed control law in (40) can achieve a better control performance. In the step (i), the matrices $\Upsilon_{i 0}, \Upsilon_{i 1}$ are tuned such that its elements on each row of $\mathbf{s}_{i}$ in (12) satisfy the Hurwitz condition. Then, the weighting gains are determined followed by Remark 3. Finally, the switching gain $\boldsymbol{\Xi}_{i}$ is determined using the trial-and-error method to achieve a desired control performance. In the step (ii), the adaptive gain matrices $\boldsymbol{\Gamma}_{0 k}, \boldsymbol{\Gamma}_{1 k}$, and $\boldsymbol{\Gamma}_{m i}$ are selected with small positive constants to achieve a better control performance compared to the CHSMC. Then, $\varepsilon$ and $\varsigma_{t}$ are tuned to avoid the chattering phenomenon. Finally, $\mathbf{K}_{\bar{\mu}}$ is tuned followed by (51) and $\mathbf{K}_{\mu}$ is tuned to reduce the effects of input saturation.

\subsection{Balancing and station-keeping control under non-zero initial lean angles}

This case study aims to validate the advantages of the proposed controller against modeled uncertainties during the balance and station-keeping task. In practice, the ballbot is not at the upright position when the rider begins to sit on. Here, the roll and pitch angles are set to $-8^{\circ}$ and $8^{\circ}$, respectively. With this configuration, the applied controllers will generate control signals as well as motor torques to keep the whole system at the upright posture and the original position, simultaneously. The ball position and body-lean angles with both CHSMC and AHSMC are recovered to its origin within around $2 \mathrm{~s}$, as shown in Figure 2(a)-(d). However, the yaw angle with the CHSMC takes a much longer settling time under uncertain model parameters and friction effects in (2). Meanwhile, with adaptive laws, the AHSMC shows its outperformance in stabilizing the yaw angle, as shown in Figure 2(e). The convergence of the sliding surfaces can be observed in Figures 2(f)-2(h), where $S_{a i}$ is the sliding surface of the AHSMC and $S_{c i}$ is the sliding surface of the CHSMC. The adaptive laws of the orientation and pose subsystems are shown in Figures 2(i) and 2(k), respectively. Here, $\hat{a}_{0 k}, \hat{a}_{1 k}$, and $\hat{a}_{2 k}$ are the elements of $\hat{\boldsymbol{\alpha}}_{i 0}, \hat{\boldsymbol{\alpha}}_{i 1}$, and $\hat{\boldsymbol{\alpha}}_{i 2}$, respectively. These estimated terms increase continuously in the transient time of $\mathbf{s}_{\Sigma}$ and remain its values when $\mathbf{s}_{\Sigma}$ converges to the origin. Figures 2(1) and 2(m) are the responses of the CHSMC and AHSMC control signals, respectively. It shows that the control signals of both controllers are saturated at the beginning. In addition, the control signals with AHSMC are quickly leaving their bounds by using the auxiliary system. This leads to the asymptotic convergence of auxiliary signals after $\breve{\boldsymbol{\tau}}=0$, as shown in Figure 2(n), according to the results in Lemma 2. From Table 3, the MAE for ball position and body-lean angles are a tradeoff. However, the RMSE and IAE of AHSMC are smaller than the ones under the CHSMC. In addition, the MAE, RMSE, and IAE of the yaw angle are much smaller under the AHSMC.

\subsection{Balancing and station-keeping control under exogenous disturbance}

In the previous scenario, model uncertainties and the variations of system parameters are considered. In this case study, we try to benchmark the system under a higher challenge. After reaching the station-keeping task from the first scenario, an exogenous disturbance affects the system via the control input $\tau_{1}$ as shown in Figure 3(1). This simulated disturbance aims to make the whole system deviates from its origin during the station-keeping operation. Figures 3(a)-3(e) describe the response of the ballbot states, showing that, by using the proposed controller, all system states have a smaller deviation and much faster recovery to the initial states. Figures $3(\mathrm{~h})$ and $3(\mathrm{k})$ are the responses of the CHSMC and AHSMC control inputs, respectively. It shows that the AHSMC takes a lower control input value to stabilize the whole system 
(Figure 3(k)) without chattering while the control signals with CHSMC surpass its bound and are saturated as shown in Figure 3(h). To improve the control performance for the CHSMC, we reconfigure the control gain $\boldsymbol{\Xi}_{1}$ to be $0.6 \times \mathbf{I}_{3}$ and denote the controller CHSMC under this configuration as CHSMC'. The tracking performance of the CHSMC' is better than the CHSMC as shown in Figures 3(a)-3(e); however, the chattering and a higher saturation phenomenon appear, as illustrated in Figure 3(i). All PIs with the AHSMC are much smaller than that of the CHSMC and CHSMC', as given in Table 3. Thus, we can conclude that the AHSMC has a significant insensitivity to the exogenous disturbance compared to the conventional HSMC.

\subsection{Stabilizing and transition control under exogenous disturbance}

In this scenario, the balance and transferring control of a ridable ballbot under exogenous disturbance is investigated. In the beginning, the ball position and the orientation angles are configured at zero values. The ballbot is commanded to transfer and balance at a new point $(1.0,1.0 \mathrm{~m})$. During the transient time, the ball moved to quadrant III to point the head toward the target, as shown in Figure 4(a). This causes the responses of the roll and pitch angles, as illustrated in Figures 4(d) and 4(e). The yaw angle is set to remain its initial angle, as in Figure 4(e). At 2s, an exogenous disturbance affects the system via $\tau_{1}$ as depicted in Figure 4(1) while the ballbot is doing the point-to-point task. As a result, the ball position and orientation angles are deviated from its tracking trajectory, as shown in Figures 4(b) and 4(c), and upright posture as shown in Figures 4(d) and 4(f). Under the applied disturbance, both the CHSMC and the AHSMC could maintain their balancing and transferring tasks. However, the AHSMC shows that it is more insensitive to external disturbances than that of the CHSMC. In other words, the actual trajectories under the control inputs of the AHSMC are almost tracked its reference signals, as shown in Figures 4(a)-4(c); the orientation angles have smaller deviations and a faster recovery to its steady states compared to the ones obtained by the CHSMC. At $5 \mathrm{~s}$, the ballbot reaches the desired target and remains in this position. The ball trajectory and the body-lean angles with the AHSMC have lower overshoots than the ones with CHSMC. Figures 4(i) and 4(k) show the response of the CHSMC and AHSMC control inputs, respectively. Also, we can observe that the control input signals with the AHSMC quickly converge to zero. As a result, the total energy consumption when applying the AHSMC is much smaller than that of the CHSMC, which is the same as the second scenario.

Remark 9. The results of the second and third scenarios show that the AHSMC is significantly insensitive to exogenous disturbance compared to the CHSMC, whether in the station-keeping or transition tasks. High deviations of ball position and body-lean angles in these scenarios can cause instability for the system operation and danger for riders when using the CHSMC in practice. In the first scenario, the CHSMC and AHSMC have a similar control performance during the transient time. However, the tracking errors with the AHSMC have smaller values compared to the ones with the CHSMC in steady time. The results in these scenarios show that the AHSMC can stabilize the system with a better control performance compared to the conventional one by using smaller gains of the switching law. Thus, chattering phenomena can be eliminated. In addition, the peaks of control signals with the AHSMC are smaller than the CHSMC. Therefore, the likelihood of the occurrence of input saturation with the AHSMC is less than the CHSMC. However, if this phenomenon happens, then the effects of actuator saturation will be reduced or avoided by the auxiliary system. In future research, mechanisms to minimize steady-state errors and steady-time under more complicated effects of uncertainties and disturbance will be investigated.

\subsection{Chattering reduction ability}

In this section, we benchmark the proposed control algorithm with higher uncertain parameters and switching gains. The damping friction coefficients are increased to as $\varsigma_{p}^{d}=4 \mathrm{~N} \cdot \mathrm{s} / \mathrm{m}, \varsigma_{b}^{d}=4 \mathrm{~N} \cdot \mathrm{m} \cdot \mathrm{s} / \mathrm{rad}$. We conduct comparative simulations between the CHSMC $\left(\boldsymbol{\Xi}_{\mathbf{1}}=0.6 \times \mathbf{I}_{3}\right)$ and two cases of the proposed algorithm with higher switching gains, i.e., AHSMC $\left(\boldsymbol{\Xi}_{\mathbf{1}}=0.6 \times \mathbf{I}_{\mathbf{3}}\right)$, and AHSMC' $\left(\boldsymbol{\Xi}_{\mathbf{1}}=1.0 \times \mathbf{I}_{3}\right)$. All system states with the AHSMC and AHSMC' have a shorter settling time, smaller steady-state errors, and lower overshoot, as 
shown in Figures 5(a)-5(e). Also, we can observe that the system state with AHSMC' has the lowest steady-state error. Even though the switching gains of the proposed algorithms are increased to greater values than the one with CHSMC, smoothed the control inputs signal are maintained with the proposed control algorithm, as shown in Figures 5(g) and 5(h). Meanwhile, the chattering phenomenon appears in control signals of the CHSMC, as shown in Figure 5(f). Therefore, the ability in reducing chattering phenomenon of the proposed control algorithm is demonstrated.

\section{CONCLUSION}

In this article, we have proposed a novel adaptive robust controller to govern the balance and transfer problems of a ridable ballbot using the FNBD under model uncertainties, external disturbance, and input saturation. The proposed controller is derived based on the HSMC scheme, which was invented for single-input-multiple-output, UMS systems. In this approach, we proposed a way to transform the FNBD into two subsystems such that the invertibility of control input matrices of the subsystems is ensured. We demonstrated that the HSMC scheme could be applied to govern the operation of the whole system by stabilizing those subsystems. The robustness of the AHSMC is demonstrated by considering the effects of model uncertainties, external disturbance, and input saturation, which are popularly happening in practical systems. In addition, using the proposed controller, the chattering phenomenon can be avoided. We carried out numerical simulations to show the advantages and robustness of the proposed algorithm in different working scenarios.

\section{FUNDING INFORMATION}

This research is partly supported by the National Research Foundation of Korea funded by the Ministry of Education (2019R1A2C2010195) and the Ministry of Science and Information and Communications Technology, Korea, under the Grand Information Technology Research Center support program (IITP-2019-2015-0-00742) supervised by the IITP. This research is also supported by the Ministry of Trade, Industry, and Energy under the Robot Industrial Core Technology Development Project program (20009214) supervised by the KEIT.

\section{REFERENCES}

[1] U. Nagarajan, G. Kantor, and R. Hollis, "The ballbot: An omnidirectional balancing mobile robot," Int. J. Rob. Res., vol. 33, no. 6, pp. 917-930, 2014, doi: 10.1177/0278364913509126.

[2] P Fankhauser; C Gwerder, "Modeling and Control of a Ballbot," Eidgenössische Technische Hochschule Zürich, 2010.

[3] K. van der Blonk, "Modeling and Control of a Ball-Balancing Robot," University of Twente, 2014.

[4] V. Do, S. Lee, and K. Gwak, "Passivity-based Nonlinear Control for a Ballbot to Balance and Transfer," Int. J. Control. Autom. Syst., vol. 17, no. 11, pp. 2929-2939, Nov. 2019, doi: 10.1007/s12555-019-0073-X.

[5] D. B. Pham, H. Kim, J. Kim, and S. G. Lee, "Balancing and Transferring Control of a Ball Segway Using a Double-Loop Approach [Applications of Control]," IEEE Control Syst., vol. 38, no. 2, pp. 15-37, Apr. 2018, doi: 10.1109/MCS.2017.2786444.

[6] K. Sukvichai and M. Parnichkun, "Double-level ball-riding robot balancing: From system design, modeling, controller synthesis, to performance evaluation," Mechatronics, vol. 24, no. 5, pp. 519-532, Aug. 2014, doi: 10.1016/j.mechatronics.2014.06.003.

[7] C. C. Tsai, M. H. Juang, C. K. Chan, C. W. Liao, and S. J. Chan, "Self-balancing and position control using multiloop approach for ball robots,” 2010 Int. Conf. Syst. Sci. Eng. ICSSE 2010, pp. 251-256, 2010, doi: 10.1109/ICSSE.2010.5551789.

[8] F. Yavuz and M. Unel, "Robust balancing and position control of a single spherical wheeled mobile 
platform," in IECON 2016 - 42nd Annual Conference of the IEEE Industrial Electronics Society, 2016, pp. 613-618, doi: 10.1109/IECON.2016.7793114.

[9] R. A. Garcia-Garcia and M. Arias-Montiel, "Linear Controllers for the NXT Ballbot with Parameter Variations Using Linear Matrix Inequalities [Lecture Notes]," IEEE Control Syst., vol. 36, no. 3, pp. 121-136, Jun. 2016, doi: 10.1109/MCS.2016.2535915.

[10] D. B. Pham, I.-S. Weon, and S.-G. Lee, "Partial Feedback Linearization Double-loop Control for a Pseudo-2D Ridable Ballbot," Int. J. Control. Autom. Syst., vol. 18, no. 5, pp. 1310-1323, May 2020, doi: 10.1007/s12555-018-0854-7.

[11] C.-H. Chiu and W.-R. Tsai, "Design and Implementation of an Omnidirectional Spherical Mobile Platform," IEEE Trans. Ind. Electron., vol. 62, no. 3, pp. 1619-1628, Mar. 2015, doi: 10.1109/TIE.2014.2360078.

[12] C. K. Chan and C. C. Tsai, "Direct adaptive recurrent interval type 2 fuzzy neural networks control using for a ball robot with a four-motor inverse-mouse ball drive," 2013 Int. Conf. Adv. Robot. Intell. Syst. ARIS 2013 - Conf. Proc., pp. 5-10, 2013, doi: 10.1109/ARIS.2013.6573525.

[13] C. E. and S. Spurgeon, Sliding Mode Control: Theory and Applications. London, U.K.: Taylor \& Francis, 1998.

[14] G. Bartolini, A. Pisano, E. Punta, and E. Usai, “A survey of applications of second-order sliding mode control to mechanical systems," Int. J. Control, vol. 76, no. 9-10, pp. 875-892, 2003, doi: 10.1080/0020717031000099010.

[15] Y. Zou, "Nonlinear robust adaptive hierarchical sliding mode control approach for quadrotors," Int. J. Robust Nonlinear Control, vol. 27, no. 6, pp. 925-941, 2017, doi: 10.1002/rnc.3607.

[16] B. Zhao, B. Xian, Y. Zhang, and X. Zhang, "Nonlinear robust sliding mode control of a quadrotor unmanned aerial vehicle based on immersion and invariance method," Int. J. Robust Nonlinear Control, vol. 25, no. 18, pp. 3714-3731, Dec. 2015, doi: 10.1002/rnc.3290.

[17] M. Van, "Adaptive neural integral sliding-mode control for tracking control of fully actuated uncertain surface vessels," Int. J. Robust Nonlinear Control, vol. 29, no. 5, pp. 1537-1557, 2019, doi: 10.1002/rnc.4455.

[18] W. Wang, J. Yi, D. Zhao, and D. Liu, "Design of a stable sliding-mode controller for a class of second-order underactuated systems," IEE Proc. - Control Theory Appl., vol. 151, no. 6, pp. 683-690, Nov. 2004, doi: 10.1049/ip-cta:20040902.

[19] N. B. Almutairi and M. Zribi, "Sliding mode control of a three-dimensional overhead crane," JVC/Journal Vib. Control, vol. 15, no. 11, pp. 1679-1730, 2009, doi: 10.1177/1077546309105095.

[20] G.-H. Kim and K.-S. Hong, “Adaptive Sliding-Mode Control of an Offshore Container Crane With Unknown Disturbances," IEEE/ASME Trans. Mechatronics, vol. 24, no. 6, pp. 2850-2861, Dec. 2019 , doi: 10.1109/TMECH.2019.2946083.

[21] L. A. Tuan, "Neural Observer and Adaptive Fractional-Order Backstepping Fast-Terminal Sliding-Mode Control of RTG Cranes," IEEE Trans. Ind. Electron., vol. 68, no. 1, pp. 434-442, Jan. 2021, doi: 10.1109/TIE.2019.2962450.

[22] L. A. Tuan, S.-G. Lee, D. H. Ko, and L. C. Nho, "Combined control with sliding mode and partial feedback linearization for 3D overhead cranes," Int. J. Robust Nonlinear Control, vol. 24, no. 18, pp. 3372-3386, Dec. 2014, doi: 10.1002/rnc.3061.

[23] R. Olfati-Saber, "Normal forms for underactuated mechanical systems with symmetry," IEEE Trans. Automat. Contr., vol. 47, no. 2, pp. 305-308, 2002, doi: 10.1109/9.983365.

[24] B. Lu, Y. Fang, and N. Sun, "Continuous Sliding Mode Control Strategy for a Class of Nonlinear Underactuated Systems,” IEEE Trans. Automat. Contr., vol. 63, no. 10, pp. 3471-3478, 2018, doi: 10.1109/TAC.2018.2794885.

[25] K. Rsetam, Z. Cao, and Z. Man, "Cascaded-Extended-State-Observer-Based Sliding-Mode Control for Underactuated Flexible Joint Robot,” IEEE Trans. Ind. Electron., vol. 67, no. 12, pp. 10822-10832, Dec. 2020, doi: 10.1109/TIE.2019.2958283.

[26] J. Huang, S. Ri, T. Fukuda, and Y. Wang, “A Disturbance Observer Based Sliding Mode Control for a 
Class of Underactuated Robotic System With Mismatched Uncertainties," IEEE Trans. Automat. Contr., vol. 64, no. 6, pp. 2480-2487, Jun. 2019, doi: 10.1109/TAC.2018.2868026.

[27] L. Chen and M. Van, "Sliding mode control of a class of underactuated system with non-integrable momentum," J. Franklin Inst., vol. 357, no. 14, pp. 9484-9504, Sep. 2020, doi: 10.1016/j.jfranklin.2020.07.022.

[28] S. ALOUI, O. PAGES, A. EL HAJJAJI, A. CHAARI, and Y. KOUBAA, "Robust Adaptive Fuzzy Sliding Mode Control Design for a class of MIMO underactuated system," IFAC Proc. Vol., vol. 44, no. 1, pp. 11127-11132, Jan. 2011, doi: 10.3182/20110828-6-IT-1002.03435.

[29] T. K. Jespersen, "Kugle - Modelling and Control of a Ball-balancing Robot," Master Thesis, 2014.

[30] D. B. Pham, J. Kim, and S.-G. Lee, "Combined control with sliding mode and Partial feedback linearization for a spatial ridable ballbot,” Mech. Syst. Signal Process., vol. 128, pp. 531-550, Aug. 2019, doi: 10.1016/j.ymssp.2019.04.008.

[31] J. Yi, W. Wang, D. Zhao, and X. Liu, "Cascade sliding-mode controller for large-scale underactuated systems,” 2005 IEEE/RSJ Int. Conf. Intell. Robot. Syst. IROS, no. 60334020, pp. 3194-3199, 2005, doi: 10.1109/IROS.2005.1545463.

[32] D. Qian, J. Yi, and D. Zhao, "Hierarchical sliding mode control for a class of SIMO under-actuated systems," Control Cybern., vol. 37, no. 1, pp. 159-175, 2008.

[33] C.-L. Hwang and W.-L. Fang, "Global Fuzzy Adaptive Hierarchical Path Tracking Control of a Mobile Robot With Experimental Validation," IEEE Trans. Fuzzy Syst., vol. 24, no. 3, pp. 724-740, Jun. 2016, doi: 10.1109/TFUZZ.2015.2476519.

[34] L. Sun and W. Huo, "Adaptive Fuzzy Control of Spacecraft Proximity Operations Using Hierarchical Fuzzy Systems,” IEEE/ASME Trans. Mechatronics, vol. 21, no. 3, pp. 1629-1640, Jun. 2016, doi: 10.1109/TMECH.2015.2494607.

[35] C. L. Hwang, C. C. Chiang, and Y. W. Yeh, "Adaptive fuzzy hierarchical sliding-mode control for the trajectory tracking of uncertain underactuated nonlinear dynamic systems," IEEE Trans. Fuzzy Syst., vol. 22, no. 2, pp. 286-299, 2014, doi: 10.1109/TFUZZ.2013.2253106.

[36] V.-T. Do and S.-G. Lee, "Neural Integral Backstepping Hierarchical Sliding Mode Control for a Ridable Ballbot Under Uncertainties and Input Saturation," IEEE Trans. Syst. Man, Cybern. Syst., pp. 1-14, 2020, doi: 10.1109/TSMC.2020.2967433.

[37] X. Zhao, H. Yang, and G. Zong, "Adaptive Neural Hierarchical Sliding Mode Control of Nonstrict-Feedback Nonlinear Systems and an Application to Electronic Circuits," IEEE Trans. Syst. Man, Cybern. Syst., vol. 47, no. 7, pp. 1394-1404, 2017, doi: 10.1109/TSMC.2016.2613885.

[38] C. Liu, Z. Zou, and T. Li, "Path following of underactuated surface vessels with fin roll reduction based on neural network and hierarchical sliding mode technique," Neural Comput. Appl., vol. 26, no. 7, pp. 1525-1535, Oct. 2015, doi: 10.1007/s00521-015-1821-3.

[39] D. B. Pham and S. G. Lee, "Hierarchical sliding mode control for a two-dimensional ball segway that is a class of a second-order underactuated system," JVC/Journal Vib. Control, vol. 25, no. 1, pp. 72-83, Jan. 2019, doi: 10.1177/1077546318770089.

[40] D. B. Pham, J. Kim, S.-G. Lee, and K.-W. Gwak, "Double-Loop Control with Hierarchical Sliding Mode and Proportional Integral Loop for 2D Ridable Ballbot," Int. J. Precis. Eng. Manuf., vol. 20 , no. 9, pp. 1519-1532, Sep. 2019, doi: 10.1007/s12541-019-00139-4.

[41] D. B. Pham and S.-G. Lee, "Aggregated Hierarchical Sliding Mode Control for a Spatial Ridable Ballbot,” Int. J. Precis. Eng. Manuf., vol. 19, no. 9, pp. 1291-1302, Sep. 2018, doi: 10.1007/s12541-018-0153-5.

[42] V.-T. Do, S.-G. Lee, and J.-H. Kim, "Robust integral backstepping hierarchical sliding mode controller for a ballbot system,” Mech. Syst. Signal Process., vol. 144, p. 106866, Oct. 2020, doi: 10.1016/j.ymssp.2020.106866.

[43] E. Pellegrini, K. J. Diepold, R. Dessort, and H. Panzer, "3D-Modeling of a Robot Balancing on a Ball," Tech. Reports Autom. Control, vol. TRAC-6, no. August 2, 2011, pp. 1-28, 2011.

[44] D. Van Thach and S. Lee, "LQG Control Design for a Coupled Ballbot Dynamical System," in 2018 
18th International Conference on Control, Automation and Systems (ICCAS), 2018, no. Iccas, pp. 666-670, doi: 978-89-93215-16-8.

[45] R. Olfati-Saber, "Global configuration stabilization for the VTOL aircraft with strong input coupling," IEEE Trans. Automat. Contr., vol. 47, no. 11, pp. 1949-1951, 2002, doi: 10.1109/TAC.2002.804457.

[46] L. A. Tuan, J. J. Kim, S. G. Lee, T. G. Lim, and L. C. Nho, "Second-order sliding mode control of a 3D overhead crane with uncertain system parameters," Int. J. Precis. Eng. Manuf., vol. 15, no. 5, pp. 811-819, 2014, doi: 10.1007/s12541-014-0404-z.

[47] M. . Zhihong and M. Palaniswami, "Robust tracking control for rigid robotic manipulators," IEEE Trans. Automat. Contr., vol. 39, no. 1, pp. 154-159, 1994, doi: 10.1109/9.273355.

[48] L. Sun and Z. Zheng, "Disturbance-Observer-Based Robust Backstepping Attitude Stabilization of Spacecraft Under Input Saturation and Measurement Uncertainty," IEEE Trans. Ind. Electron., vol. 64, no. 10, pp. 7994-8002, Oct. 2017, doi: 10.1109/TIE.2017.2694349.

[49] G. Tao, "A simple alternative to the Barbalat lemma," IEEE Trans. Automat. Contr., vol. 42, no. 5, p. 698, May 1997, doi: 10.1109/9.580878. 


\section{APPENDIX A: THE TERMS IN THE SYSTEM MATRICES}

$$
\begin{aligned}
& c_{x}=\cos (\varphi), c_{y}=\cos (\theta), c_{z}=\cos (\psi), c_{1}=c_{\varphi} c_{\theta} c_{\psi}, c_{2}=c_{\varphi}^{2} c_{\theta} c_{\psi}^{2}, c_{3}=c_{\varphi}^{2} c_{\theta}^{2}, c_{4}=c_{\varphi}^{2} c_{\psi}^{2}, c_{x z}=c_{\varphi}^{2} c_{\psi}^{2}, c_{x y}=c_{\varphi}^{2} c_{\theta}^{2}, \\
& I_{w r}=I_{w} /\left(4 r_{w}^{2}\right), I_{w c}=I_{w r} / 2, I_{b 1}=I_{b x}+I_{b y}-I_{b z}, I_{b 2}=8 I_{b z}-I_{b y}, \\
& \dot{p}_{x y}=\dot{p}_{x}-\dot{p}_{y}, \dot{p}_{y x}=\dot{p}_{x}+\dot{p}_{y}, \dot{\theta}_{1}=\dot{\theta}-\dot{\varphi}, \dot{\theta}_{2}=\dot{\psi}+\dot{\varphi} \text {, } \\
& m_{1}=3\left(c_{1}^{2}-c_{x y}-2 c_{2}-2 c_{x z}+c_{\varphi}^{2}+c_{\psi}^{2}+1\right) I_{w r}+\left(m_{b}+m_{s}+I_{s} / r_{s}^{2}\right) \text {, } \\
& m_{2}=3\left(c_{1}^{2}+2 c_{2}-c_{\varphi}^{2} c_{\theta}-2 c_{x z}+c_{\psi}^{2}\right) I_{w r}, \\
& m_{3}=3\left(c_{\varphi}^{2} c_{\psi} r_{s}-2 c_{\psi} r_{s}+c_{\varphi}^{2} c_{\theta} c_{\psi} r_{s}\right) I_{w r}+c_{\varphi} c_{\theta} c_{\psi} l m_{b}, \\
& m_{4}=-3 c_{\theta} c_{\psi} r_{s} I_{w r}+\left(c_{\varphi} c_{\psi}-c_{1}\right) l m_{b} \text {, } \\
& m_{5}=3\left(c_{\varphi}^{2} c_{\theta} c_{\psi} r_{s}-c_{x y} c_{\psi} r_{s}\right) I_{w r}+\left(c_{\varphi} c_{\psi}-c_{1}\right) l m_{b}, \\
& m_{6}=3\left(2 c_{2}-c_{1}^{2}+2 c_{x z}-c_{\varphi}^{2}-c_{\psi}^{2}+2\right) I_{w r}+\left(m_{b}+m_{s}+I_{s} / r_{s}^{2}\right) \text {, } \\
& m_{7}=3\left(c_{\varphi}^{2} c_{\psi} r_{s}-2 c_{\psi} r_{s}-c_{\varphi}^{2} c_{\theta} c_{\psi} r_{s}\right) I_{w r}+c_{1} l m_{b} \text {, } \\
& m_{8}=3 c_{\theta} c_{\psi} r_{s} I_{w r}-c_{\varphi} c_{\psi} l m_{b}-c_{1} l m_{b} \text {, } \\
& m_{9}=3 c_{\varphi} c_{\theta} r_{s}\left(c_{\varphi} c_{\psi}+c_{1}\right) I_{w r}+\operatorname{lm}_{b}\left(c_{\varphi} c_{\psi}+c_{1}\right) \text {, } \\
& m_{10}=\left(3 c_{\varphi}^{2} r_{s}^{2}+3 r_{s}^{2}\right) I_{w r}+\left(I_{b z} c_{\varphi}^{2}-I_{b y} c_{\varphi}^{2}-m_{b} c_{\varphi}^{2} l^{2}+m_{b} l^{2}+I_{b y}\right), \\
& m_{11}=-3 c_{\varphi}^{2} c_{\theta} r_{s}^{2} I_{w r}+c_{\varphi}^{2} c_{\theta}\left(m_{b} l^{2}-I_{b z}+I_{b y}\right) \text {, } \\
& m_{12}=3 r_{s}^{2} I_{w r}+\left(m_{b} l^{2}+I_{b x}\right) \text {, } \\
& m_{13}=-3 c_{\theta} r_{s}^{2} I_{w r}-c_{\theta}\left(m_{b} l^{2}+I_{b x}\right) \text {, } \\
& m_{14}=\left(3 c_{x y} r_{s}^{2}+3 r_{s}^{2}\right) I_{w r}+\left(I_{b z} c_{x y}-I_{b y} c_{x y}-m_{b} c_{x y} l^{2}-I_{b x} c_{\theta}^{2}+I_{b y} c_{\theta}^{2}+m_{b} l^{2}+I_{b x}\right), \\
& c_{11}=6 I_{w c}\left[c_{3} \dot{\theta}+\left(2 c_{4}+c_{\varphi}^{2} c_{\theta}-c_{\psi}^{2}\right) \dot{\psi}+\left(c_{3}+2 c_{4}+c_{\theta} c_{\psi}^{2}-c_{\varphi}^{2}\right) \dot{\varphi}-\left(\dot{\theta}_{2}+\dot{\theta}\right) c_{1}^{2}-(2 \dot{\psi}+\dot{\theta}+2 \dot{\varphi}) c_{2}\right] \text {, } \\
& \left.c_{12}=-3 I_{w c}\left[\left(c_{x y}+c_{2 z} c_{2 x}\right) \dot{\psi}+\left(c_{\theta} c_{2 x}-4 c_{x z}+2 c_{\theta} c_{\psi}^{2}\right) \dot{\varphi}+c_{\varphi}^{2} c_{\theta} \dot{\theta}\right)+2(\dot{\theta}-\dot{\psi}+\dot{\varphi}) c_{1}^{2}-2(\dot{\theta}-2 \dot{\psi}+2 \dot{\varphi}) c_{2}\right] \text {, } \\
& c_{13}=\left(6 I_{w c} c_{\varphi} r_{s}\left(\dot{\theta}+\dot{\varphi}-c_{\theta} \dot{\psi}\right)-8 l_{m}\left(\dot{\theta}_{2}+\dot{\theta}\right)\right) c_{1}+6 I_{w c}\left\{\left(\dot{p}_{x} c_{\theta}^{2}-\dot{p}_{y} c_{\theta} / 2-c_{\psi} r_{s} \dot{\varphi}\right) c_{\varphi}^{2}+\left[c_{\psi} r_{s} \dot{\psi}-\dot{p}_{y x} c_{1}^{2}-\dot{p}_{x y} c_{2}\right]\right\} \text {, } \\
& c_{14}=I_{w c} 6\left[c_{\varphi} r_{s}\left(\dot{\psi}+\dot{\theta}+c_{\theta} \dot{\psi}\right) c_{1}-\dot{p}_{y x} c_{1}^{2}-2 \dot{p}_{x y} c_{2}\right]+l_{m} c_{\varphi} c_{\psi}(\dot{\psi}-8 \dot{\varphi})-l_{m}(8 \dot{\theta}-\dot{\psi}+8 \dot{\varphi}) c_{1} \\
& +I_{w c}\left[3 c_{\theta} \dot{p}_{y}-3 \dot{p}_{x} s_{2 x}+12 c_{x z} \dot{p}_{y x}-6 c_{\varphi}^{2}\left(c_{\theta} \dot{p}_{y}-c_{\theta}^{2} \dot{p}_{x}+c_{\psi} r_{s} \dot{\theta}\right)+6 c_{\theta} c_{\psi}^{2} \dot{p}_{x y}-6 c_{\theta} c_{\psi} r_{s} \dot{\psi}\right] \text {, } \\
& c_{15}=I_{w c}\left[3\left(c_{3}+4 c_{4}-c_{2 x}-2 c_{\psi}^{2}\right) \dot{p}_{y}+3\left(s_{2 z}-4 c_{4}-6 c_{\varphi}^{2} c_{\theta}\right) \dot{p}_{x}+6 c_{\psi} r_{s}\left(c_{\theta} \dot{\varphi}-\dot{\theta}\right)\right] \\
& -I_{w c} 6 \dot{p}_{x y} c_{1}^{2}-I_{w c} 12 \dot{p}_{y x} c_{2}-\left\{I_{w c} 6\left[c_{\varphi} r_{s}(\dot{\psi}-\dot{\varphi})+c_{\varphi} c_{\theta} r_{s}\left(\dot{\psi}+\dot{\theta}_{1}\right)\right]+l_{m}(8 \dot{\psi}+8 \dot{\theta}-\dot{\varphi})\right\} c_{1}-l_{m} c_{\varphi} c_{\psi}(8 \dot{\psi}-\dot{\varphi}) \text {, } \\
& c_{21}=6 I_{w c}\left\{(\dot{\theta}-2 \dot{\psi}+2 \dot{\varphi}) c_{2}-(\dot{\theta}-\dot{\psi}+\dot{\varphi}) c_{1}^{2}-\left[\left(c_{3}+2 c_{\psi}^{2} c_{2 x}-c_{2 x}\right) \dot{\psi}+\left(c_{2 x} c_{\theta}+2 c_{\theta} c_{\psi}^{2}-4 c_{4}\right) \dot{\varphi}+c_{\varphi}^{2} c_{\theta} \dot{\theta}\right] / 2\right\} \text {, } \\
& c_{22}=I_{w c}\left\{6\left(\dot{\theta}_{2}+\dot{\theta}\right) c_{1}^{2}+6(2 \dot{\psi}+\dot{\theta}+2 \dot{\varphi}) c_{2}-\left[\left(c_{2 x} c_{\psi}^{2}+c_{\varphi}^{2} c_{\theta}\right) \dot{\psi}+6\left(c_{\theta}+c_{2 x}\right) c_{\psi}^{2} \dot{\varphi}\right]\right\} \text {, } \\
& c_{23}=-\left[I_{w c} 6 c_{\varphi} r_{s}\left(\dot{\theta}+\dot{\varphi}-c_{\theta} \dot{\psi}\right)+l_{m}(8 \dot{\theta}-\dot{\psi}+8 \dot{\varphi})\right] c_{1}-6 I_{w c}\left[\dot{p}_{x y} c_{1}^{2}+\dot{p}_{y x} c_{2}-\left(c_{\psi} r_{s} \dot{\psi}+c_{\varphi}^{2} c_{\theta} \dot{p}_{x} / 2+c_{\varphi}^{2} c_{\psi} r_{s} \dot{\varphi}\right)\right] \text {, } \\
& c_{24}=-I_{w c} 6 c_{\varphi} r_{s}\left(\dot{\theta}-\dot{\psi}+c_{\theta} \dot{\psi}\right)-I_{w c} 6 \dot{p}_{x y} c_{1}^{2}-8 l_{m}\left(\dot{\theta}_{2}+\dot{\theta}\right) c_{1}+l_{m} c_{\varphi} c_{\psi} \dot{\theta}_{2}+12 I_{w c} \dot{p}_{y x} c_{2} \\
& -3 I_{w c}\left[\left(2 c_{\theta} c_{\psi}^{2}-s_{2 x}\right) \dot{p}_{y}+\left(2 c_{\varphi}^{2} c_{\theta}+c_{\theta} c_{2 z}\right) \dot{p}_{x}-4 c_{x z} \dot{p}_{x y}+2 c_{\psi} r_{s}\left(c_{\theta} \dot{\psi}+c_{\varphi}^{2} \dot{\theta}\right)\right] \text {, } \\
& c_{25}=\left\{6 c_{\varphi} r_{s} I_{w c}\left[\left(1-c_{\theta}\right) \dot{\theta}_{2}+c_{\theta} \dot{\theta}\right]-l_{m}\left(8 \dot{\theta}_{2}-\theta+8 \dot{\varphi}\right)\right\} c_{1}+6 I_{w c} \dot{p}_{y x} c_{1}^{2}-I_{w c} 12 \dot{p}_{x y} c_{2} \\
& +l_{m} c_{\varphi} c_{\psi} \dot{\theta}_{2}-3 I_{w c}\left[\left(c_{x y}+c_{2 x} s_{\psi}^{2}\right) \dot{p}_{x}+\left(2 c_{x z}-s_{2 z}+2 c_{\varphi}^{2} c_{\theta}\right) \dot{p}_{y}+2 c_{\psi} r_{s}\left(\dot{\theta}+c_{\theta} \dot{\varphi}\right)\right] \text {, } \\
& c_{31}=6 I_{w c}\left\{c_{\varphi} r_{s}\left(\dot{\psi}+c_{\theta} \dot{\psi}+\dot{\varphi}\right) c_{1}+\dot{p}_{y x} c_{1}^{2}+\dot{p}_{x y} c_{2}-c_{\varphi}^{2} c_{\theta}\left(c_{\theta} \dot{p}_{x}-\dot{p}_{y} / 2\right)-c_{\psi}\left[c_{\varphi}^{2} r_{s} \dot{\theta}_{2}+r_{s}\left(c_{\theta} \dot{\varphi}-\dot{\psi}\right)\right]\right\}, \\
& c_{32}=6 I_{w c}\left[\dot{p}_{x y} c_{1}^{2}-c_{\varphi} r_{s}\left(\dot{\varphi}-\dot{\psi}+c_{\theta} \dot{\psi}\right) c_{1}-\dot{p}_{y x} c_{2}+c_{\varphi}^{2} c_{\theta} \dot{p}_{x} / 2+c_{\psi} r_{s}\left(c_{\theta} \dot{\varphi}-s_{\varphi}^{2} \dot{\psi}-c_{\varphi}^{2} \dot{\varphi}\right)\right] \text {, } \\
& c_{33}=\dot{\varphi}\left[3 r_{s}^{2} s_{2 x} I_{w c}-4 s_{2 x} l_{b}-s_{2 x}\left(8 I_{b y}-I_{b z}\right) / 2\right] \text {, }
\end{aligned}
$$




$$
\begin{aligned}
& \left.c_{34}=\left(c_{\varphi}^{2} c_{\theta} \dot{\psi}-4 s_{2 x} \dot{\theta}\right) l_{b}-I_{w c}\left\{6 c_{\psi} r_{s}\left[c_{\theta} \dot{p}_{x y}+c_{\varphi}^{2} \dot{p}_{y x}\right]-3 r_{s}^{2} s_{2 x} \dot{\theta}-6 c_{\theta} r_{s}^{2} s_{\varphi}^{2} \dot{\psi}\right)\right\} \\
& +4\left(I_{b x}-I_{b y}+I_{b z}\right) c_{\theta} \dot{\psi}+\left(I_{b y}-8 I_{b z}\right) c_{\varphi}^{2} c_{\theta} \dot{\psi}+\left(I_{b z} / 2-4 I_{b y}+\right) s_{2 x} \dot{\theta}+I_{w c} 6 c_{\varphi} \dot{p} r_{s} \dot{p}_{x y} c_{1} \text {, } \\
& c_{35}=I_{w c} 6 r_{s}\left\{c_{\varphi}\left[\dot{p}_{y x}+c_{\theta} \dot{p}_{x y}\right] c_{1}+c_{\theta} r_{s}\left(s_{\varphi}^{2} \dot{\varphi}+c_{\varphi}^{2} c_{\theta} \dot{\psi}\right)+c_{\psi} s_{\varphi}^{2} \dot{p}_{x y}\right\}+c_{\varphi}^{2} c_{\theta}\left(\dot{\varphi}-8 c_{\theta} \dot{\psi}\right) l_{b} \\
& +\left(4 I_{b x}+I_{b y}\left(c_{\varphi}^{2}-4\right)-4 I_{b z} c_{2 x}\right) c_{\theta} \dot{\varphi}+\left(I_{b y} s_{2 y} / 2-8 I_{b y} c_{x y}+I_{b z} c_{x y}-4 I_{b x} s_{2 y}\right) \dot{\psi}, \\
& c_{41}=I_{w c} 6\left\{\dot{p}_{y x} c_{1}^{2}-c_{\varphi} r_{s}\left(\dot{\psi}+c_{\theta} \dot{\psi}+\dot{\theta}\right) c_{1}+2 \dot{p}_{x y} c_{2}-\left[2 c_{4} \dot{p}_{y x}+c_{\theta} c_{\psi}^{2} \dot{p}_{x y}-c_{\varphi}^{2} s_{\theta}^{2} \dot{p}_{x}-c_{\psi} r_{s} \dot{\theta}\left(c_{\theta}+c_{\varphi}^{2}\right)\right]+c_{2 x} c_{\theta} \dot{p}_{y} / 2\right\} \text {, } \\
& c_{42}=I_{w c} 6\left\{c_{\varphi} r_{s}\left(\dot{\theta}-\dot{\psi}+c_{\theta} \dot{\psi}\right) c_{1}+\dot{p}_{x y} c_{1}^{2}-2 \dot{p}_{y x} c_{2}+\left[c_{2} c_{\theta} \dot{p}_{x} / 2-c_{\varphi}^{2} \dot{p}_{y}-2 c_{4} \dot{p}_{x y}+c_{\theta} c_{\psi}^{2} \dot{p}_{y x}+c_{\psi} r_{s}\left(c_{\varphi}^{2}-c_{\theta}\right) \dot{\theta}\right]\right\} \text {, } \\
& c_{43}=-I_{w c} 6 c_{\varphi} r_{s} \dot{p}_{x y} c_{1}+\left(s_{2 x} \dot{\theta} / 2-8 c_{\varphi}^{2} c_{\theta} \dot{\psi}\right) l_{b}+I_{w c} 3 r_{s}\left[2 c_{\theta} c_{\psi} \dot{p}_{x y}+3 c_{\varphi}^{2} c_{\psi} \dot{p}_{y x}+I_{b z} c_{\varphi}^{2} c_{\theta} \dot{\psi}\right. \\
& \left.-r_{s}\left(s_{2 x} \dot{\theta}+c_{2 x} c_{\theta} \dot{\psi}\right)\right]+4 c_{\theta}\left(I_{b y}-I_{b z}-I_{b x}\right) \dot{\psi}+\left(I_{b y} / 2-4 I_{b z}\right) s_{2 x} \dot{\theta}-8 I_{b y} c_{\varphi}^{2} c_{\theta} \dot{\psi}, \\
& c_{45}=-I_{w c} 6 c_{\varphi} c_{\theta} r_{s} c_{1} \dot{p}_{x y}-8 c_{\varphi}^{2} c_{\theta}\left(\dot{\theta}+c_{\theta} \dot{\psi}_{x}\right) l_{b}-6 I_{w c} c_{\theta} r_{s}\left[c_{\varphi}^{2} c_{\psi} \dot{p}_{y x}+r_{s}\left(s_{\varphi}^{2} \dot{\theta}-c_{\varphi}^{2} c_{\theta} \dot{\psi}_{x}\right)\right] \\
& -c_{\theta}\left[4\left(I_{b x}+I_{b z}-I_{b y}\right) \dot{\theta}+8 c_{\varphi}^{2}\left(I_{b y}-I_{b z}\right)\left(\dot{\theta}+c_{\theta} \dot{\psi}_{x}\right)\right] \text {, } \\
& c_{51}=3 I_{w c}\left[2 \dot{p}_{x y} c_{1}^{2}+4 \dot{p}_{y x} c_{2}-2 c_{\varphi} r_{s}\left(1+c_{\theta}\right) \dot{\theta}_{1} c_{1}+\left(\dot{p}_{x} s_{2 z}-c_{2 z} \dot{p}_{y}-2 c_{\varphi}^{2}\left(\dot{p}_{y}+c_{\theta} \dot{p}_{x}\right)+c_{x y} \dot{p}_{y}-4 c_{x z} \dot{p}_{x y}-2 s_{\varphi}^{2} c_{\psi} r_{s} \dot{\theta}\right)\right] \text {, } \\
& c_{52}=3 I_{w c}\left[\left(c_{3} \dot{p}_{x}-c_{2 z} \dot{p}_{x}-\dot{p}_{y} s_{2 z}-2 c_{\varphi}^{2}\left(\dot{p}_{x}-c_{\theta} \dot{p}_{y}\right)+4 c_{4} \dot{p}_{y x}+2 s_{\varphi}^{2} c_{\psi} r_{s} \dot{\theta}\right)-2 c_{\varphi} r_{s}\left[\dot{\theta}_{1}\left(1-c_{\theta}\right)\right] c_{1}-2 \dot{p}_{y x} c_{1}^{2}+4 \dot{p}_{x y} c_{2}\right] \text {, } \\
& c_{53}=-I_{w c} 6 c_{\varphi} r_{s}\left(\dot{p}_{y x}+c_{\theta} \dot{p}_{x y}\right) c_{1}+\left(c_{3} \dot{\psi}_{x}-8 c_{\theta} \dot{\varphi}-8 c_{\varphi}^{2} c_{\theta} \dot{\theta}+c_{\varphi}^{2} c_{\theta} \dot{\varphi}\right) l_{b} \\
& -I_{w c}\left[6 c_{\psi} s_{\varphi}^{2} r_{s} \dot{p}_{x y}+6 c_{\varphi}^{2} c_{\theta} r_{s}^{2}\left(c_{\theta} \dot{\psi}_{x}-\dot{\theta}_{1}\right)\right]-4 c_{\theta} I_{b 1} \dot{\varphi}-c_{\varphi}^{2} c_{\theta} I_{b 2} \dot{\varphi} \\
& +\left(I_{b x} / 2-4 I_{b y}\right) s_{2 y} \dot{\psi}_{x}-I_{b 2} c_{3} \dot{\psi}_{x}+c_{\varphi}^{2} c_{\theta}\left(I_{b z}-8 I_{b y}\right) \dot{\theta} \text {, } \\
& c_{54}=6 I_{w c} c_{\varphi} c_{\theta} r_{s}\left[\dot{p}_{x y} c_{1}-c_{\varphi}\left(r_{s} \dot{\theta}-c_{\psi} \dot{p}_{y x}+c_{\theta} r_{s} \dot{\psi}\right)\right]+c_{\theta}\left(c_{\varphi}^{2} \dot{\theta}-8 \dot{\theta}+c_{\varphi}^{2} c_{\theta} \dot{\psi}\right) l_{b}-c_{\theta}\left[4 I_{b 1} \dot{\theta}+c_{\varphi}^{2} I_{b 2}\left(\dot{\theta}+c_{\theta} \dot{\psi}\right)\right] \text {, } \\
& c_{55}=c_{\theta}^{2}\left\{\left(I_{b x}-8 I_{b y}\right) \dot{\theta}+c_{\varphi}^{2}(\dot{\theta}+\dot{\varphi})\left[\left(I_{b y}-8 I_{b z}\right)+l_{b}-6 r_{s}^{2} I_{w c}\right]\right\} \text {, } \\
& b_{11}=-\sqrt{2}\left[\sqrt{2}\left(c_{\varphi} c_{\psi}-c_{1}\right) / 2+\sqrt{2} c_{\theta} c_{\psi} / 2\right] / r_{s} \text {, } \\
& b_{12}=-\left(c_{\varphi} c_{\psi}-c_{\theta} c_{\psi} / 2+\sqrt{3} c_{\varphi} c_{\psi} / 2-c_{1}+\sqrt{3} c_{1} / 2\right) / r_{s} \text {, } \\
& b_{13}=\left(c_{\theta} c_{\psi} / 2-c_{\varphi} c_{\psi}+\sqrt{3} c_{\varphi} c_{\psi} / 2+c_{1}+\sqrt{3} c_{1} / 2\right) / r_{s} \text {, } \\
& b_{21}=-\left[\left(c_{\varphi} c_{\psi}+c_{1}\right)-c_{\theta} c_{\psi}\right] / r_{s} \text {, } \\
& b_{22}=-\left(c_{\varphi} c_{\psi}+c_{\theta} c_{\psi} / 2+\sqrt{3} c_{\varphi} c_{\psi} / 2+c_{1}-\sqrt{3} c_{1} / 2\right) / r_{s} \text {, } \\
& b_{23}=-\left(c_{\varphi} c_{\psi}+c_{\theta} c_{\psi} / 2-\sqrt{3} c_{\varphi} c_{\psi} / 2+c_{1}+\sqrt{3} c_{1} / 2\right) / r_{s} \text {, } \\
& b_{31}=c_{\varphi} \text {, } \\
& b_{32}=c_{\varphi}+\sqrt{3} c_{\varphi} / 2 \text {, } \\
& b_{33}=c_{\varphi}-\sqrt{3} c_{\varphi} / 2 \text {, } \\
& b_{41}=1, b_{42}=-1 / 2, b_{43}=-1 / 2 \text {, } \\
& b_{51}=-c_{\theta}-c_{\varphi} c_{\theta} \text {, } \\
& b_{52}=c_{\theta} / 2-c_{\varphi} c_{\theta}+\sqrt{3} c_{\varphi} c_{\theta} / 2 \text {, } \\
& b_{53}=c_{\theta} / 2-c_{\varphi} c_{\theta}-\sqrt{3} c_{\varphi} c_{\theta} / 2 \text {, } \\
& g_{3}=-\operatorname{lm}_{b} g c_{\varphi} s_{\theta}, g_{4}=-\operatorname{lm}_{b} g c_{\theta} s_{\varphi} \text {. }
\end{aligned}
$$




\section{APPENDIX B: PROOF OF THE INVERTIBLE PROPERTY OF THE TRANSFORMED INPUT} MATRICES

Recall from the dynamic transformation, we have

$$
\mathbf{B}_{0}=\mathbf{B}_{b 1}-\mathbf{M}_{b p 1} \mathbf{M}_{p 1}^{-1} \mathbf{B}_{p 1}, \mathbf{B}_{1}=\mathbf{B}_{p 2}-\mathbf{M}_{p b 2} \mathbf{M}_{b 2}^{-1} \mathbf{B}_{b 2} .
$$

The determinant of these matrices are determined as

$$
\begin{aligned}
\operatorname{det}\left(\mathbf{B}_{0}\right) & =\frac{6 \sqrt{6} c_{\theta} r_{s}^{3} r_{w}\left[I_{s}+\left(m_{b}+m_{s}\right) r_{s}^{2}+c_{\varphi} c_{\theta} l m_{b} r_{s}\right]^{2}}{\left[4 I_{s} r_{w}^{2}+3 I_{w} r_{s}^{2}+4\left(m_{b}+m_{s}\right) r_{s}^{2} r_{w}^{2}\right]\left[4 I_{s} r_{w}^{2}+\left(m_{b}+m_{s}\right) r_{s}^{2} r_{w}^{2}+3 I_{w} r_{s}^{2}\left(2-c_{\varphi}^{2} c_{\theta}^{2}\right)\right]}, \\
\operatorname{det}\left(\mathbf{B}_{1}\right) & =-6 \sqrt{6} c_{\theta} r_{s} r_{w} N_{b 1} / D_{b 1},
\end{aligned}
$$

where

$$
\begin{aligned}
N_{b 1}= & I_{b x} I_{b z} s_{\varphi}^{2} c_{\theta}+I_{b x} I_{b y} c_{\varphi}^{2} c_{\theta}+\left(l^{2}+r_{s}^{2}\right) c_{\varphi}^{2} c_{\theta} l^{2} m_{b}^{2}+\left(1+c_{\varphi}^{2} c_{\theta}^{2}\right) c_{\varphi} l^{3} m_{b}^{2} r_{s} \\
& +\left[I_{b z} s_{\varphi}^{2}+\left(I_{b x}+I_{b y}\right) c_{\varphi}^{2}\right] c_{\theta} l^{2} m_{b}+\left(I_{b x} c_{\theta}^{2}+I_{b y} s_{\theta}^{2}+I_{b z} s_{\theta}^{2} c_{\theta}^{2}+I_{b y} c_{\varphi}^{2} c_{\theta}^{2}\right) c_{\varphi} l m_{b} r_{s}, \\
D_{b 1}= & \left(4 m_{b} l^{2} r_{w}^{2}+3 I_{w} r_{s}^{2}+4 I_{b x} r_{w}^{2}\right)\left[4 I_{b y} c_{\varphi}^{2} r_{w}^{2}+4 I_{b z} s_{\varphi}^{2} r_{w}^{2}+3\left(1+s_{\varphi}^{2}\right) I_{w} r_{s}^{2}+4 c_{\varphi}^{2} l^{2} m_{b} r_{w}^{2}\right] .
\end{aligned}
$$

It is notice that during the operation of ballbot the lean angle of the body is constrained as (6) From (6) and (55), we can conclude that

$$
\operatorname{det}\left(\mathbf{B}_{0}\right)>0, \operatorname{det}\left(\mathbf{B}_{1}\right)<0 .
$$

Thus, the invertibility of $\mathbf{B}_{0}$ and $\mathbf{B}_{1}$ matrices is guaranteed. Q.E.D.

\section{APPENDIX C: PROOF OF LEMMA 1}

Let $\chi=\left[\begin{array}{llll}\chi_{1} & \chi_{2} & \cdots & \chi_{n}\end{array}\right]^{T},(9)$ can be rewritten as follows.

$$
\sum_{i=1}^{n} \chi_{i} \tanh \left(\chi_{i} / \varepsilon\right)=\sum_{i=1}^{n} \chi_{i} \frac{\sinh \left(\chi_{i} / \varepsilon\right)}{\cosh \left(\chi_{i} / \varepsilon\right)}
$$

Using the Maclaurin series for the hyperbolic sine and cosine functions, we have

$$
\left\{\begin{array}{rl}
\chi_{i} \sinh \left(\chi_{i} / \varepsilon\right) & =\chi_{i} \sum_{n=0}^{\infty} \frac{\chi_{i}^{2 n+1}}{\varepsilon^{2 n+1}(2 n+1) !} \geq 0 \\
\cosh \left(\chi_{i} / \varepsilon\right) & =\sum_{n=0}^{\infty} \frac{\chi_{i}^{2 n}}{\varepsilon^{2 n}(2 n) !}>0
\end{array}, \varepsilon>0, \chi_{i} \in \mathfrak{R}^{n} .\right.
$$

From (58) and (59) we obtain

$$
\chi^{T} \tanh (\chi / \varepsilon) \geq 0, \forall \varepsilon>0, \chi \in \mathfrak{R}^{n}
$$

Q.E.D. 


\section{APPENDIX D: PROOF OF LEMMA 2}

If $\mathbf{s}_{\Sigma}$ converges to zero as $t \rightarrow \infty$, then all three rows of $\mathbf{s}_{\Sigma}$ in (20) asymptotically converge to zero. As a result, $s_{\psi}$ will asymptotically converge to zero. In addition, if the sliding surfaces of the first level of the hierarchy are defined as in (12) and are constructed at the second layer as in (13). Also, if the weighting gains of $\boldsymbol{\Gamma}_{s i}$ is selected according to the rule in (21), then the pairs of sliding surfaces $\left(s_{x}, s_{\theta}\right)$ and $\left(s_{y}, s_{\varphi}\right)$ will asymptotically converge to zero by Theorem 2 in [42]. Since the tracking error vectors are constructed as (12) , where $\Upsilon_{i 0}, \Upsilon_{i 1}$ satisfy the Hurwitz condition, then the asymptotic convergence of these vectors can be achieved. Q.E.D. 
TABLE 1

Parameters of the ridable ballbot

\begin{tabular}{|c|l|l|}
\hline Symbol & \multicolumn{1}{|c|}{ Parameter } & \multicolumn{1}{|c|}{ Value } \\
\hline$m_{b}$ & Body mass & $116 \mathrm{~kg}$ \\
\hline$l$ & Body length & $0.23 \mathrm{~m}$ \\
\hline$I_{b x}$ & Moment of inertia of the body about the roll axis & $16.25 \mathrm{~kg} \cdot \mathrm{m}^{2}$ \\
\hline$I_{b y}$ & Moment of inertia of the body about the pitch axis & $15.85 \mathrm{~kg} \cdot \mathrm{m}^{2}$ \\
\hline$I_{b z}$ & Moment of inertia of the body about the yaw axis & $1.08 \mathrm{~kg} \cdot \mathrm{m}^{2}$ \\
\hline$r_{w}$ & OW radius & $0.1 \mathrm{~m}$ \\
\hline$I_{w}$ & Moment of inertia of the OW & $0.26 \mathrm{~kg} \cdot \mathrm{m}^{2}$ \\
\hline$m_{s}$ & Ball mass & $11.4 \mathrm{~kg}$ \\
\hline$r_{s}$ & Ball radius & $0.19 \mathrm{~m}$ \\
\hline$I_{s}$ & Moment of inertia of the ball & $0.165 \mathrm{~kg} \cdot \mathrm{m}^{2}$ \\
\hline
\end{tabular}

TABLE 2

Control parameters

\begin{tabular}{|c|c|c|c|}
\hline$\Upsilon_{00}$ & $\Upsilon_{01}$ & $\overline{\boldsymbol{\Gamma}}_{0}$ & $\boldsymbol{\Xi}_{0}$ \\
\hline $\operatorname{diag}(1,1,1)$ & $\operatorname{diag}(5,5,3)$ & $5 \times \mathbf{I}_{\mathbf{3}}$ & $5.6 \times \mathbf{I}_{3}$ \\
\hline$\Upsilon_{10}$ & $\Upsilon_{11}$ & $\overline{\boldsymbol{\Gamma}}_{1}$ & $\boldsymbol{\Xi}_{1}$ \\
\hline $\operatorname{diag}(1,1,1)$ & $\operatorname{diag}(2.3,2.3,3)$ & $6.8 \times \operatorname{diag}(1,-1,1)$ & $0.01 \times \mathbf{I}_{3}$ \\
\hline
\end{tabular}

TABLE 3

Performance comparisons

\begin{tabular}{|c|c|c|c|c|c|c|c|c|c|c|c|}
\hline \multirow[b]{2}{*}{ PI } & \multirow{2}{*}{ Controllers } & \multicolumn{5}{|c|}{ Case Study I } & \multicolumn{5}{|c|}{ Case Study II } \\
\hline & & $p_{x}(\mathrm{~m})$ & $p_{y}(\mathrm{~m})$ & $\varphi\left(^{0}\right)$ & $\theta\left({ }^{0}\right)$ & $\psi\left({ }^{\circ}\right)$ & $p_{x}(\mathrm{~m})$ & $p_{y}(\mathrm{~m})$ & $\varphi\left(^{0}\right)$ & $\theta\left({ }^{0}\right)$ & $\psi\left({ }^{\circ}\right)$ \\
\hline \multirow{3}{*}{ MAE } & CHSMC & 0.183 & 0.157 & 5.981 & 4.489 & 1.18 & 0.238 & 0.181 & 14.101 & 10.472 & 36.645 \\
\hline & CHSMC' & \multicolumn{5}{|c|}{-} & 0.236 & 0.178 & 14.031 & 10.469 & 36.431 \\
\hline & AHSMC & 0.185 & 0.150 & 6.330 & 4.431 & 0.586 & 0.183 & 0.152 & 6.235 & 4.541 & 10.319 \\
\hline \multirow{3}{*}{ RMSE } & CHSMC & 0.048 & 0.041 & 1.876 & 1.449 & 0.387 & 0.057 & 0.039 & 3.078 & 2.107 & 5.967 \\
\hline & CHSMC' & \multicolumn{5}{|c|}{-} & 0.054 & 0.038 & 2.959 & 2.043 & 5.877 \\
\hline & AHSMC & 0.047 & 0.039 & 1.882 & 1.405 & 0.136 & 0.025 & 0.019 & 0.978 & 0.649 & 0.818 \\
\hline \multirow{3}{*}{ ITAE } & CHSMC & 22.49 & 17.89 & 1015.3 & 717.87 & 361.4 & 80.704 & 54.081 & 4315.81 & 2877.65 & 5916.10 \\
\hline & CHSMC' & \multicolumn{5}{|c|}{-} & 70.10 & 46.50 & 3800.60 & 2516.96 & 5388.01 \\
\hline & AHSMC & 20.74 & 16.57 & 969.34 & 661.24 & 67.42 & 25.544 & 17.10 & 1264.84 & 815.41 & 474.53 \\
\hline
\end{tabular}

Tables 1, 2, and 3 